\title{
Elimination of Skolem functions for monotone formulas in analysis
}

\section{Ulrich Kohlenbach}

Department of Mathematics, University of Michigan, Ann Arbor, MI 48109, USA, e-mail: kohlenb@math.lsa.umich.edu

The date of receipt and acceptance will be inserted by the editor

\section{Introduction}

In [14] we have introduced a hierarchy $\left(\mathrm{G}_{n} \mathrm{~A}^{\omega}\right)_{n \in \mathbb{N}}$ of subsystems of arithmetic in all finite types where the growth of the definable functions of $\mathrm{G}_{n} \mathrm{~A}^{\omega}$ corresponds to the well-known Grzegorczyk hierarchy. For certain (in general) non-constructive analytical axioms $\Delta$ and the schema of quantifier-free choice AC-qf the following rule is shown:

¿From a proof

$$
\mathrm{G}_{n} \mathrm{~A}^{\omega}+\Delta+\mathrm{AC} \text {-qf } \vdash \forall u^{1}, k^{0} \forall v \leq_{\tau} t u k \exists w^{\gamma} A_{0}(u, k, v, w),
$$

(where $t$ is a closed term, $A_{0}$ is quantifier-free and contains only $u, k, v, w$ free, $\gamma \leq 2, \rho$ is an arbitrary type and $\leq_{\tau}$ is defined pointwise) one can extract (by monotone functional interpretation) a uniform bound $\Phi$ on $\exists w^{\gamma}$ which is given by a closed term of $\mathrm{G}_{n} \mathrm{~A}^{\omega}$ and does not depend on $v$, i.e.

$$
\forall u, k \forall v \leq t u k \exists w \leq \Phi u k A_{0}(u, k, v, w)
$$

holds in the full set-theoretic model.

In particular $\Phi u k$ is a polynomial (an elementary recursive function) in $u^{M}:=\lambda x^{0} \cdot \max _{i \leq x} u(i)$ and $k^{0}$ in case $n=2$ (resp. $\left.n=3\right)$.

In a paper under preparation we will show that substantial parts of classical analysis can be carried out in $\mathrm{G}_{3} \mathrm{~A}^{\omega}+\Delta+\mathrm{AC}$-qf and even in $\mathrm{G}_{2} \mathrm{~A}^{\omega}+\Delta+\mathrm{AC}$-qf for suitable $\Delta$ (see [14] for more information on this).

On the other hand there are central theorems in analysis whose proofs use arithmetical instances of $\mathrm{AC}$, i.e. instances of

$$
\mathrm{AC}_{a r}: \forall x^{0} \exists y^{0} A(x, y) \rightarrow \exists f^{1} \forall x^{0} A(x, f x),
$$


where $A \in \Pi_{\infty}^{0}$ ( $A$ may contain parameters of arbitrary type), and which are not covered by the results mentioned above.

Examples are the following theorems

1. The principle of convergence for bounded monotone sequences of real numbers (or equivalently: every bounded monotone sequence of reals has a Cauchy modulus (PCM)).

2. For every sequence of real numbers which is bounded from above there exists a least upper bound.

3. The Bolzano-Weierstraß property for bounded sequences in $\mathbb{R}^{d}$ (for every fixed $d$ ).

4. The Arzelà-Ascoli lemma.

5. The existence of the limit superior for bounded sequences of real numbers.

We will investigate these theorems (w.r.t. to their contribution to the rate of growth of uniform bounds extractable from proofs which use them) in a subsequent paper using the method developed in this paper and discuss now only (PCM) in order to motivate the results of the present paper which is the second one in a sequence of papers resulting from the authors Habilitationsschrift [12]. All undefined notions are used in the sense of [14] on which this paper relies. $A_{0}, B_{0}, C_{0}, \ldots$ always denote quantifier-free formulas.

Using a convenient representation of real numbers, $(\mathrm{PCM})$ can be formalized as follows:

$$
(\mathrm{PCM}):\left\{\begin{aligned}
\forall a_{(\cdot)}^{1(0)}, & , c^{1}\left(\forall n^{0}\left(c \leq \mathbb{R} a_{n+1} \leq_{\mathbb{R}} a_{n}\right)\right. \\
& \left.\rightarrow \exists h^{1} \forall k^{0} \forall m, \tilde{m} \geq_{0} h k\left(\left|a_{m}-\mathbb{R}_{\mathbb{R}} a_{\tilde{m}}\right| \leq_{\mathbb{R}} \frac{1}{k+1}\right)\right) .
\end{aligned}\right.
$$

(PCM) immediately follows from its arithmetical weakening

$$
\left(\mathrm{PCM}^{-}\right):\left\{\begin{aligned}
\forall a_{(\cdot)}^{1(0)} & , c^{1}\left(\forall n^{0}\left(c \leq \mathbb{R} a_{n+1} \leq_{\mathbb{R}} a_{n}\right)\right. \\
& \left.\rightarrow \forall k^{0} \exists n^{0} \forall m, \tilde{m} \geq_{0} n\left(\left|a_{m}-\mathbb{R} a_{\tilde{m}}\right| \leq_{\mathbb{R}} \frac{1}{k+1}\right)\right)
\end{aligned}\right.
$$

by an application of $\mathrm{AC}_{a r}$ to

$$
A: \equiv \forall m, \tilde{m} \geq n\left(\left|a_{m}-\mathbb{R} a_{\tilde{m}}\right| \leq_{\mathbb{R}} \frac{1}{k+1}\right) \in \Pi_{1}^{0}
$$

$\left(\leq_{\mathbb{R}} \in \Pi_{1}^{0}\right.$ follows from the fact that real numbers are given as Cauchy sequences of rationals with fixed rate of convergence in our theories).

It is well-known that a constructive functional interpretation of the negative translation of $\mathrm{AC}_{a r}$ requires so-called bar-recursion and cannot be caried out e.g. in Gödel's term calculus $T$ (see [21] and [15] ). $\mathrm{AC}_{a r}$ is (using classical logic) equivalent to $\mathrm{CA}_{a r}+\mathrm{AC}^{0,0}-\mathrm{qf}$, where

$$
\mathrm{CA}_{a r}: \exists g^{1} \forall x^{0}\left(g(x)={ }_{0} 0 \leftrightarrow A(x)\right) \text { with } A \in \Pi_{\infty}^{0},
$$

(and $\mathrm{AC}^{0,0}-\mathrm{qf}$ is the restriction of $\mathrm{AC}_{a r}$ to quantifier-free formulas) and therefore causes an immense rate of growth (when added to e.g. $\mathrm{G}_{2} \mathrm{~A}^{\omega}$ ). ¿From the work in the context of 'reverse mathematics' (see e.g. [3],[20]) it is known that 1)-5) imply CA $\mathrm{CA}_{a r}$ relatively to (a second-order version of) $\widehat{\mathrm{PA}}^{\omega} \uparrow+\mathrm{AC}^{0,0}$-qf (see [1] for the definition of $\widehat{\mathrm{PA}}^{\omega} \wedge$ ). In [12] it is shown that this holds even relatively to $\mathrm{G}_{2} \mathrm{~A}^{\omega}$. 
In contrast to these general facts we prove in this paper a meta-theorem which in particular implies that if (PCM) is applied in a proof only to sequences $\left(a_{n}\right)$ which are given explicitely in the parameters of the proposition (which is proved) then this proof can be (effectively) transformed (without causing new growth) into a proof of the same conclusion which uses only $\left(\mathrm{PCM}^{-}\right)$for these sequences. By this transformation the use of $\mathrm{AC}_{a r}$ is eliminated and the determination of the growth caused (potentially by $(\mathrm{PCM})$ ) reduces to the determination of the growth caused by $\left(\mathrm{PCM}^{-}\right)$.

More precisely our meta-theorem has the following consequence:

Let $\mathcal{T}^{\omega}:=\mathrm{G}_{n} \mathrm{~A}^{\omega}$ and $\chi$ denote a closed term of $\mathrm{G}_{n} \mathrm{~A}^{\omega}$ (having an appropriate type). Then the following rule holds

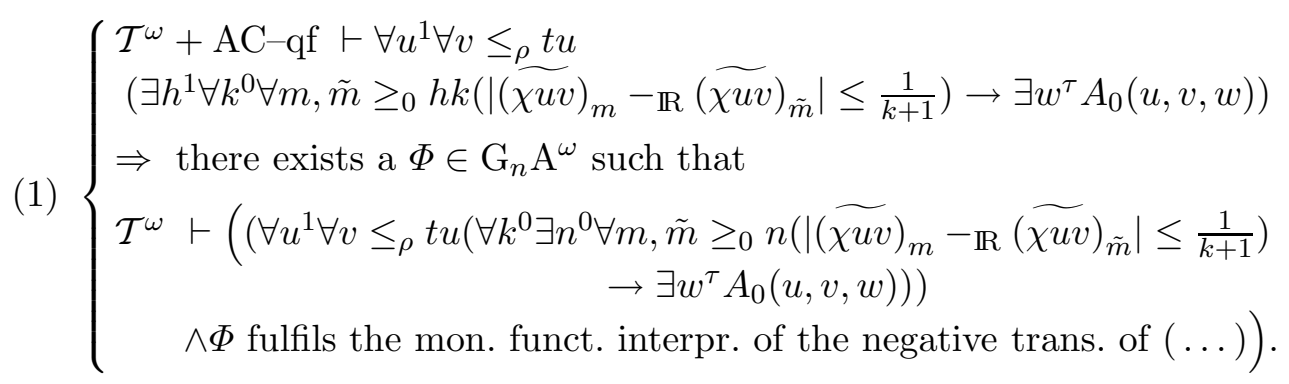

$\left(\right.$ Here $\tilde{a}(n):=\max _{\mathbb{R}}\left(0, \min _{i \leq n}(a(i))\right)$. By this construction every sequence $a^{1(0)}$ represents a decreasing sequence of positive real numbers. The restriction to the special lower bound $c:={ }_{\mathbb{R}} 0$ is convenient but of course not essential.)

In contrast to $(\mathrm{PCM})$ the (negative translation of the) principle $\left(\mathrm{PCM}^{-}\right)$has a simple constructive monotone functional interpretation which is fulfilled by a functional $\Psi$ which is primitive recursive in the sense of [6]. Because of the nice behaviour of the monotone functional interpretation with respect to the modus ponens one obtains (by applying $\Phi$ to $\Psi)$ a monotone functional interpretation of

$$
\forall u^{1} \forall v \leq_{\rho} t u \exists w^{\tau} A_{0}(u, v, w)
$$

and so (if $\tau \leq 2$ ) using tools from [11],[14] a uniform bound $\xi$ for $\exists w$, i.e.

$$
\forall u^{1} \forall v \leq_{\rho} t u \exists w \leq_{\tau} \xi u A_{0}(u, v, w),
$$

where $\xi$ is primitive recursive in the sense of Kleene [6] (and not only in the generalized sense of Gödel's calculus $T$ ).

This conclusion also holds for sequences of instances $\forall n^{0} \operatorname{PCM}(\chi u v n)$ of $\operatorname{PCM}(a)$ instead of $\operatorname{PCM}(\chi u v)$.

Let us consider the following general situation:

For

$$
F(\underline{a}): \equiv \forall x_{1}^{0} \exists y_{1}^{0} \ldots \forall x_{k}^{0} \exists y_{k}^{0} F_{0}\left(x_{1}, y_{1}, \ldots, x_{k}, y_{k}, \underline{a}\right),
$$

where $\underline{x}, \underline{y}, \underline{a}$ are all the free variables of $F_{0}$, we define the Skolem normal form $F^{S}$ of $F$ by

$$
F^{S}(\underline{a}): \equiv \exists f_{1}, \ldots, f_{k} \forall x_{1}^{0}, \ldots, x_{k}^{0} F_{0}\left(x_{1}, f_{1} x_{1}, \ldots, x_{k}, f_{k} x_{1} \ldots x_{k}, \underline{a}\right) .
$$


If we could prove that

$$
\text { (2) }\left\{\begin{array}{l}
\mathcal{T}^{\omega}(+\mathrm{AC}-\mathrm{qf}) \vdash \forall u^{1} \forall v \leq_{\rho} t u\left(F^{S}(u, v) \rightarrow \exists w^{\tau} A_{0}(u, v, w)\right) \Rightarrow \\
\mathcal{T}^{\omega} \vdash \forall u^{1} \forall v \leq_{\rho} t u\left(F(u, v) \rightarrow \exists w^{\tau} A_{0}(u, v, w)\right),
\end{array}\right.
$$

then (1) would follow as a special case.

(2) in turn is implied by

$$
\text { (3) } \mathcal{T}^{\omega}(+\mathrm{AC}-\mathrm{qf}) \vdash G^{H} \Rightarrow \mathcal{T}^{\omega} \vdash G,
$$

where

$$
G^{H}: \equiv\left\{\begin{array}{r}
\forall u^{1} \forall v \leq_{\rho} t u \forall h_{1}, \ldots, h_{k} \exists y_{1}^{0}, \ldots, y_{k}^{0}, w^{\tau} \\
G_{0}\left(u, v, y_{1}, h_{1} y_{1}, y_{2}, h_{2} y_{1} y_{2}, \ldots, y_{k}, h_{k} y_{1} \ldots y_{k}, w\right)
\end{array}\right.
$$

is the (generalized $)^{1}$ Herbrand normal form of

$$
G: \equiv \forall u^{1} \forall v \leq_{\rho} t u \exists y_{1}^{0} \forall x_{1}^{0} \ldots \exists y_{k}^{0} \forall x_{k}^{0} \exists w^{\tau} G_{0}\left(u, v, y_{1}, x_{1}, \ldots, y_{k}, x_{k}, w\right) .
$$

Since $\forall u^{1} \forall v \leq_{\rho} t u\left(F(u, v) \rightarrow \exists w^{\tau} A_{0}\right)$ can be transformed into a prenex normal form $G$ whose Herbrand normal form is logically equivalent to

$\forall u \forall v \leq t u\left(F^{S}(u, v) \rightarrow \exists w A_{0}\right),(2)$ is a special case of (3).

Unfortunately (3) is wrong (even without AC-qf) for $\mathcal{T}^{\omega}=\mathrm{G}_{n} \mathrm{~A}^{\omega}, \mathrm{PRA}^{\omega}$ and much weaker theories. In fact it is false already for the first-order fragments of these theories augmented by function variables. For (a second-order fragment of) $\mathrm{PRA}^{\omega}+\Sigma_{1}^{0}-\mathrm{IA}$ this was proved firstly in [10] (thereby detecting a false argument in the literature). In $\S 2$ below we will prove a result which implies this as a special case and refutes (3) also for $\mathrm{G}_{n} \mathrm{~A}^{\omega}$ (and their second-order fragments even when the universal axioms 9) from the definition of $\mathrm{G}_{n} \mathrm{~A}^{\omega}$ are replaced by the schema of quantifier-free induction).

On the other hand we will show that (3) is true for $\mathcal{T}^{\omega}=\mathrm{G}_{n} \mathrm{~A}^{\omega}$ (but remains false for $\mathcal{T}^{\omega}=\mathrm{PRA}^{\omega}$ ) if $G$ satisfies a certain monotonicity condition (see def.26 below) which is fulfilled e.g. in (1). We may add also axioms $\Delta$ to $\mathrm{G}_{n} \mathrm{~A}^{\omega}$ having the form $\forall x^{\delta} \exists y \leq_{\tau} s x \forall z^{\gamma} G_{0}(x, y, z)$, where $\mathrm{G}_{0}$ is quantifier-free and $s$ a closed term. As mentioned above such axioms cover substantial parts of classical analysis relatively to $\mathrm{G}_{2} \mathrm{~A}^{\omega}$ (see [12] and [14] for details).

This result will be used in $\S 3$ to determine the growth caused by (sequences of) instances of the restriction of $\mathrm{AC}_{a r}$ and $\mathrm{CA}_{a r}$ to $\Pi_{1}^{0}$ formulas: $\Pi_{1}^{0}-\mathrm{AC}, \Pi_{1}^{0}-\mathrm{CA}$.

In a subsequent paper we will treat the analytical principles mentioned above. It will turn out that 1)-4) have the same contribution to the growth of uniform bounds as $\Pi_{1}^{0}$-CA, whereas 5) may produce a growth of the Ackermann type.

1 The Herbrand normal form is usually defined only for arithmetical formulas, i.e. if $u, v, w$ are not present. In this case it coincides with our definition. In $\mathrm{G}_{2} \mathrm{~A}^{+}$in $\S 2$ below, $u, v, v$ do not occur and the $h_{i}$ are free function variables. 
2 Elimination of Skolem functions of type $0(0) \ldots(0)$ in higher type theories for monotone formulas: no additional growth

We first prove a result which in particular refutes (3) from the introduction (even without AC-qf) for $\mathrm{G}_{n} \mathrm{~A}^{\omega}$ (with $n \geq 2$ ), $\mathrm{G}_{\infty} \mathrm{A}^{\omega}$ and $\mathrm{PRA}^{\omega}$ :

Let $\mathrm{G}_{2} \mathrm{~A}$ be the first-order part of $\mathrm{G}_{2} \mathrm{~A}^{\omega}$ (without the universal axioms 9) from [14] but only with the schema of quantifier-free induction instead of them) and $G_{2} A^{+}$be $G_{2} A$ augmented by function variables and a substitution rule

$$
S U B: \frac{A(f)}{A(g)} \text {. }
$$

$\mathrm{G}_{2} \mathrm{~A}^{+}$contains the schema of quantifier-free induction with function parameters .

Proposition 21 Let $A \in \Pi_{\infty}^{0}$ be a theorem of (first-order) Peano arithmetic PA. Then one can construct a sentence $\tilde{A} \in \Pi_{\infty}^{0}$ such that

$$
G_{2} A^{+} \vdash \tilde{A}^{H} \text { and } G_{2} A \vdash A \leftrightarrow \tilde{A} .
$$

Proof: If PA $\vdash A$, then there are arithmetical instances (without function parameters) of the induction schema such that for their universal closure $\tilde{F}_{1}, \ldots, \tilde{F}_{k}$

$$
\mathrm{G}_{2} \mathrm{~A} \vdash \bigwedge_{i=1}^{k} \tilde{F}_{i} \rightarrow A,
$$

since PA $\subset \mathrm{G}_{2} \mathrm{~A}+\Pi_{\infty}^{0}-\mathrm{IA}^{-}$, where $\Pi_{\infty}^{0}-\mathrm{IA}^{-}$is the induction schema for all arithmetical formulas without function variables.

Let $B$ be any prenex normal form of $\left(\bigwedge_{i=1}^{k}\left(y_{i}={ }_{0} 0 \leftrightarrow F_{i}\left(x_{i}\right)\right) \rightarrow A\right)$, where $F_{i}$ denotes the induction formula of $\tilde{F}_{i}$, then

$$
\tilde{A}: \equiv \exists \underline{a}, x_{1}, \ldots, x_{k} \forall y_{1}, \ldots, y_{k} B\left(x_{1}, \ldots, x_{k}, y_{1}, \ldots, y_{k}, \underline{a}\right)
$$

is a prenex normal form of

$$
\forall \underline{a}, x_{1}, \ldots, x_{k} \exists y_{1}, \ldots, y_{k} \bigwedge_{i=1}^{k}\left(y_{i}=0 \leftrightarrow F_{i}\left(x_{i}\right)\right) \rightarrow A,
$$

where $\underline{a}$ are the (number) parameters of the induction formulas $F_{i}$. Because of

$$
\mathrm{G}_{2} \mathrm{~A} \vdash \forall \underline{a}, x_{1}, \ldots, x_{k} \exists y_{1}, \ldots, y_{k} \bigwedge_{i=1}^{k}\left(y_{i}=0 \leftrightarrow F_{i}\left(x_{i}\right)\right),
$$

we obtain

$$
\mathrm{G}_{2} \mathrm{~A} \vdash A \leftrightarrow \tilde{A}
$$


Since $\tilde{A}^{H}$ is logically implied by

$$
C: \equiv \exists \underline{a}, x_{1}, \ldots, x_{k} B\left(x_{1}, \ldots, x_{k}, f_{1} \underline{a} x_{1} \ldots x_{k}, \ldots, f_{k} \underline{a} x_{1} \ldots x_{k}, \underline{a}\right),
$$

it remains to show that $\mathrm{G}_{2} \mathrm{~A}^{+} \vdash C$ :

Assume $\forall \underline{a}, x_{1}, \ldots, x_{k} \bigwedge_{i=1}^{k}\left(f_{i} \underline{a} x_{1} \ldots x_{k}=0 \leftrightarrow F_{i}\left(x_{i}\right)\right)$. Quantifier-free induction applied to $A_{0}\left(x_{i}\right): \equiv\left(f_{i}\left(\underline{a}, 0, \ldots, 0, x_{i}, 0, \ldots, 0\right)=0\right)$ yields $\tilde{F}_{i}$. Hence

$$
\mathrm{G}_{2} \mathrm{~A}^{+} \vdash \forall \underline{a}, x_{1}, \ldots, x_{k} \bigwedge_{i=1}^{k}\left(f_{i} \underline{a} x_{1} \ldots x_{k}=0 \leftrightarrow F_{i}\left(x_{i}\right)\right) \rightarrow A,
$$

i.e. $\mathrm{G}_{2} \mathrm{~A}^{+} \vdash C$.

Corollary 22 (to the proof) Let $G_{2} A\left[f_{1}, \ldots, f_{k}\right]$ denote the extension of $G_{2} A$ which is obtained by adding new function symbols $f_{1}, \ldots, f_{k}$ which may occur in instances of QF-IA. Then $G_{2} A\left[f_{1}, \ldots, f_{k}\right] \vdash \tilde{A}^{H}$ and $G_{2} A \vdash A \leftrightarrow \tilde{A}$ (with $A, \tilde{A}$ as in the proof above), where $f_{1}, \ldots, f_{k}$ are the function symbols used in the definition of $\tilde{A}^{H}$.

Corollary 231 . For each $n \in \mathbb{N}$ one can construct a sentence $A \in \Pi_{\infty}^{0}$ such that

$$
G_{2} A^{\omega} \vdash A^{H} \text {, but } G_{\infty} A^{\omega}+\Sigma_{n}^{0}-I A \subset P R A^{\omega}+\Sigma_{n}^{0}-I A \nvdash A .
$$

2. For each $n \in \mathbb{N}$ one can construct sentences $A \in \Pi_{\infty}^{0}$ and a sentence $\forall x^{0} \exists y^{0} B_{0}(x, y) \in \Pi_{2}^{0}$ such that

$$
G_{2} A^{\omega} \vdash A^{H} \text {, but } G_{2} A^{\omega}+A \vdash \forall x^{0} \exists y^{0} B_{0}(x, y),
$$

where $f x:=\min y\left[B_{0}(x, y)\right]$ is not $<\omega_{n}(\omega)$-recursive.

Proof: 1) Let $n \geq 1$ and $A \in \mathcal{L}(\mathrm{PA})$ be an instance of $\Sigma_{n+1}^{0}$-IA which is not provable in $\mathrm{PRA}^{\omega}+\Sigma_{n}^{0}$-IA (such an instance exists since every $<\omega_{n+1}(\omega)$-recursive function is provably recursive in $\mathrm{PRA}^{\omega}+\Sigma_{n+1}^{0}$-IA, but in $\mathrm{PRA}^{\omega}+\Sigma_{n}^{0}$-IA only $<\omega_{n}(\omega)$-recursive functions are provably recursive (This follows from [18](thm.5) using the fact that PRA ${ }^{\omega}+\Sigma_{n}^{0}$-IA has a functional interpretation by functionals in Parsons calculus $\left.T_{n-1}\right)$ and there are $<\omega_{n+1}(\omega)-$ recursive functions which are not $<\omega_{n}(\omega)$-recursive). Construct now $\tilde{A}$ as in prop.21. It follows that $\mathrm{G}_{2} \mathrm{~A}^{\omega} \vdash \tilde{A}^{H}$, but PRA ${ }^{\omega}+\Sigma_{n}^{0}-\mathrm{IA} \nvdash \tilde{A}$.

2) follows from prop.21 and the fact that every $\alpha\left(<\varepsilon_{0}\right)$-recursive function is provably recursive in $\mathrm{PA}$.

The reason for the provability of $\tilde{A}^{H}$ in prop.21 is that the schema of quantifier-free induction is applicable to the index functions used in defining $\tilde{A}^{H}$. This always is the case in the presence of the substitution rule $S U B$ or $\forall^{1}$-elimination in theories like $\mathrm{G}_{2} \mathrm{~A}^{\omega}$ where quantification over functions is possible. 
In the following we show that the same phenomenon occurs if $\mathrm{QF}-\mathrm{IA}$ in $\mathrm{G}_{2} \mathrm{~A}^{+}$is restricted to formulas without function variables but instead of this new functional symbols $\Phi_{\max , n}$ are added (for each number $n \in \mathbb{N}$ ) together with the axioms

$$
(\max , n): \bigwedge_{i=1}^{n}\left(y_{i} \leq_{0} x_{i}\right) \rightarrow f \underline{y} \leq_{0} \Phi_{\max , n} f \underline{x},
$$

where $f$ is an $n$-ary function variable.

$$
(\max ):=\cup_{n}(\max , n) .
$$

We call the resulting system $\mathrm{G}_{2} \mathrm{~A}+(\max )$.

Remark 24 (max, 1$)$ is fulfilled by the functional $\Phi_{1} f x=\max (f 0, \ldots, f x)$ from $G_{n} A^{\omega}$. By $\lambda$-abstraction and finite iteration of $\Phi_{1}$ one can easily define a functional satisfying (max, $n$ ) (Hence $G_{2} A+(\max )$ is a subsystem of $\left.G_{2} A^{\omega}\right)$. This is the reason for calling this axiom (max). Of course instead of $\Phi_{1}$ one could also use e.g. $\Phi_{2} f x=\sum_{i=0}^{x} f i$.

Proposition 25 Let $A \in \Pi_{\infty}^{0}$ be a theorem of PA. Then one can construct a sentence $\tilde{A} \in \Pi_{\infty}^{0}$ such that

$$
G_{2} A+(\max ) \vdash \tilde{A}^{H} \text { and } G_{2} A \vdash A \leftrightarrow \tilde{A} .
$$

Proof: Since PA $\vdash A$, there are arithmetical instances (without function parameters) of the induction schema such that for their universal closure $\tilde{F}_{1}, \ldots, \tilde{F}_{k}$

$$
\mathrm{G}_{2} \mathrm{~A} \vdash \bigwedge_{i=1}^{k} \tilde{F}_{i} \rightarrow A .
$$

Lets consider now the so-called collection principle

$$
\mathbf{C P}: \forall \tilde{x}^{0}\left(\forall x<_{0} \tilde{x} \exists y^{0} F(x, y, \underline{a}) \rightarrow \exists z \forall x<_{0} \tilde{x} \exists y<_{0} z F(x, y, \underline{a})\right),
$$

where $x, y, \underline{a}$ are all free variables of $F$. This principle has been studied proof-theoretically in [17] and also in [19]. By [19] (prop.4.1 (iv)) one can construct for every instance $\tilde{F}$ of $\Sigma_{n}^{0}$-IA instances $F_{i}$ of $\Sigma_{n+1}^{0}-\mathrm{CP}$ (i.e. CP restricted to $\Sigma_{n+1}^{0}$-formulas) such that $\bigwedge_{i} F_{i} \rightarrow \tilde{F}$. From the proof in [19] (which uses only QF-IA and the function - ) it follows that $\mathrm{G}_{2} \mathrm{~A} \vdash \wedge F_{i} \rightarrow \tilde{F}$. Let $F_{1}, \ldots, F_{l}$ denote such instances of $\mathrm{CP}$ whose universal closures imply $\tilde{F}_{1}, \ldots, \tilde{F}_{k} . F_{i}$ has the form

$$
F_{i}: \equiv\left(\forall x<{ }_{0} \tilde{x} \exists y^{0} G_{i}(x, y, \underline{a}) \rightarrow \exists z \forall x<\tilde{x} \exists y<z G_{i}(x, y, \underline{a})\right) .
$$

Thus $\mathrm{G}_{2} \mathrm{~A}$ proves

$$
\text { (1) } \forall \underline{a}, \tilde{x} \bigwedge_{i=1}^{l}\left(\forall x_{i}<_{0} \tilde{x} \exists y_{i}^{0} G_{i}\left(x_{i}, y_{i}, \underline{a}\right) \rightarrow \exists z_{i} \forall x_{i}<\tilde{x} \exists y_{i}<z_{i} G_{i}\left(x_{i}, y_{i}, \underline{a}\right)\right) \rightarrow A .
$$


Consider now

$$
B: \equiv\left\{\begin{array}{l}
\forall \underline{a}, \tilde{x}, x_{1}, \ldots, x_{l} \exists y_{1}, \ldots, y_{l} \\
\bigwedge_{i=1}^{\wedge}\left(\forall u_{i}<\tilde{x} \exists w_{i} G_{i}\left(u_{i}, w_{i}, \underline{a}\right) \rightarrow\left(x_{i}<\tilde{x} \rightarrow G_{i}\left(x_{i}, y_{i}, \underline{a}\right)\right)\right) \rightarrow A
\end{array}\right.
$$

and

$$
C: \equiv\left(\bigwedge_{i=1}^{l}\left(\forall u_{i}<\tilde{x} \exists w_{i} G_{i}\left(u_{i}, w_{i}, \underline{a}\right) \rightarrow\left(x_{i}<\tilde{x} \rightarrow G_{i}\left(x_{i}, y_{i}, \underline{a}\right)\right)\right) \rightarrow A\right) .
$$

Let $C^{p r}$ be an (arbitrary) prenex normal form of $C$. Then

$$
B^{p r}: \equiv \exists \underline{a}, \tilde{x}, x_{1}, \ldots, x_{l} \forall y_{1}, \ldots, y_{l} C^{p r}\left(\tilde{x}, x_{1}, \ldots, x_{l}, y_{1}, \ldots, y_{l}, \underline{a}\right)
$$

is a prenex normal form of $B$.

We now show i) $\mathrm{G}_{2} \mathrm{~A}+(\max ) \vdash\left(B^{p r}\right)^{H}$ and ii) $\mathrm{G}_{2} \mathrm{~A} \vdash B^{p r} \leftrightarrow A$.

i) Define

$$
\widehat{B}: \equiv \exists \underline{a}, \tilde{x}, x_{1}, \ldots, x_{l} C^{p r}\left(\tilde{x}, x_{1}, \ldots, x_{l}, f_{1} \underline{a} \tilde{x} x_{1} \ldots x_{l}, \ldots, f_{l} \underline{a} \tilde{x} x_{1} \ldots x_{l}, \underline{a}\right) .
$$

The implication $\widehat{B} \rightarrow\left(B^{p r}\right)^{H}$ holds logically. Hence we have to show that $\mathrm{G}_{2} \mathrm{~A}+(\max ) \vdash \widehat{B}$ : $\widehat{B}$ is logically equivalent to

$$
\text { (2) } \forall \underline{a}, \tilde{x} \bigwedge_{i=1}^{l}(\underbrace{\forall u_{i}<\tilde{x} \exists w_{i} G_{i} \rightarrow \forall \underline{x}\left(x_{i}<\tilde{x} \rightarrow G_{i}\left(x_{i}, f_{i} \underline{a} \tilde{x} x_{1} \ldots x_{l}, \underline{a}\right)\right)}_{H_{i}: \equiv}) \rightarrow A .
$$

By $(\max )$ applied to $f_{i}, \forall x_{i}\left(x_{i}<\tilde{x} \rightarrow G_{i}\left(x_{i}, f_{i} \underline{a} \tilde{x} x_{1} \ldots x_{l}, \underline{a}\right)\right)$ implies $\exists z_{i} \forall x_{i}<\tilde{x} \exists y_{i}<z_{i} G_{i}\left(x_{i}, y_{i}, \underline{a}\right)$. Thus

$$
\mathrm{G}_{2} \mathrm{~A}+(\max ) \vdash H_{i} \rightarrow F_{i} \text { for } i=1, \ldots l .
$$

By (1),(2) this yields $\mathrm{G}_{2} \mathrm{~A}+(\max ) \vdash \widehat{B}$.

ii) We have to show that $\mathrm{G}_{2} \mathrm{~A} \vdash B \leftrightarrow A$. This follows immediately from the fact that

$$
\forall \underline{a}, \tilde{x}, x_{1}, \ldots, x_{l} \exists y_{1}, \ldots, y_{l} \bigwedge_{i=1}^{l}\left(\forall u_{i}<\tilde{x} \exists w_{i} G_{i}\left(u_{i}, w_{i}, \underline{a}\right) \rightarrow\left(x_{i}<\tilde{x} \rightarrow G_{i}\left(x_{i}, y_{i}, \underline{a}\right)\right)\right)
$$

holds logically.

Prop. 21 and prop. 25 show that for theories like $\mathrm{G}_{n} \mathrm{~A}^{\omega}$ the Herbrand normal form $A^{H}$ of an arithmetical formula $A$ in general is much weaker than $A$ with respect to provability in $\mathrm{G}_{n} \mathrm{~A}^{\omega}$ (compare cor.23). This phenomenon does not occur if $A$ satisfies the following monotonicity condition: 
Definition 26 Let $A \in \mathcal{L}\left(G_{n} A^{\omega}\right)$ be a formula having the form

$$
A \equiv \forall u^{1} \forall v \leq_{\tau} t u \exists y_{1}^{0} \forall x_{1}^{0} \ldots \exists y_{k}^{0} \forall x_{k}^{0} \exists w^{\gamma} A_{0}\left(u, v, y_{1}, x_{1}, \ldots, y_{k}, x_{k}, w\right),
$$

where $A_{0}$ is quantifier-free and contains only $u, v, \underline{y}, \underline{x}, w$ free. Furthermore let $t$ be $\in G_{n} R^{\omega}$ and $\tau, \gamma$ are arbitrary finite types.

1. A is called (arithmetically) monotone if

$$
\operatorname{Mon}(A): \equiv\left\{\begin{array}{r}
\forall u^{1} \forall v \leq_{\tau} t u \forall x_{1}, \tilde{x}_{1}, \ldots, x_{k}, \tilde{x_{k}}, y_{1}, \tilde{y}_{1}, \ldots y_{k}, \tilde{y}_{k} \\
\left(\bigwedge_{i=1}^{k}\left(\tilde{x}_{i} \leq_{0} x_{i} \wedge \tilde{y}_{i} \geq_{0} y_{i}\right) \wedge \exists w^{\gamma} A_{0}\left(u, v, y_{1}, x_{1}, \ldots, y_{k}, x_{k}, w\right)\right. \\
\left.\rightarrow \exists w^{\gamma} A_{0}\left(u, v, \tilde{y}_{1}, \tilde{x}_{1}, \ldots, \tilde{y}_{k}, \tilde{x}_{k}, w\right)\right) .
\end{array}\right.
$$

2. The Herbrand normal form $A^{H}$ of $A$ is defined to be

$$
\begin{aligned}
A^{H}: \equiv \forall u^{1} \forall v \leq_{\tau} t u \forall h_{1}^{\rho_{1}}, \ldots, h_{k}^{\rho_{k}} \exists y_{1}^{0}, \ldots, y_{k}^{0}, w^{\gamma} \\
\underbrace{A_{0}\left(u, v, y_{1}, h_{1} y_{1}, \ldots, y_{k}, h_{k} y_{1} \ldots y_{k}, w\right)}_{A_{0}^{H}: \equiv}, \text { where } \rho_{i}=0 \underbrace{0(0) \ldots(0)}_{i} .
\end{aligned}
$$

Theorem 27 Let $n \geq 1$ and $\Psi_{1}, \ldots, \Psi_{k} \in G_{n} R^{\omega}$. Then

$$
\begin{aligned}
G_{n} A^{\omega}+\operatorname{Mon}(A) \vdash \forall & u^{1} \forall v \leq_{\tau} t u \forall h_{1}, \ldots, h_{k}\left(\bigwedge_{i=1}^{k}\left(h_{i} \text { monotone }\right)\right. \\
& \left.\rightarrow \exists y_{1} \leq_{0} \Psi_{1} u \underline{h} \ldots \exists y_{k} \leq_{0} \Psi_{k} u \underline{h} \exists w^{\gamma} A_{0}^{H}\right) \rightarrow A,
\end{aligned}
$$

where $\left(h_{i}\right.$ monotone) $: \equiv \forall x_{1}, \ldots, x_{i}, y_{1}, \ldots, y_{i}\left(\bigwedge_{j=1}^{i}\left(x_{j} \geq_{0} y_{j}\right) \rightarrow h_{i} \underline{x} \geq_{0} h_{i} \underline{y}\right)$.

Theorem 28 Let $A$ be as in thm.27 and $\Delta$ be as in [14](thm.3.2.2), i.e. a set of sentences $\forall x^{\delta} \exists y \leq_{\rho} s x \forall z^{\eta} G_{0}(x, y, z)$ where $s$ is a closed term of $G_{n} A^{\omega}$ and $G_{0}$ a quantifier-free formula, and let $A^{\prime}$ denote the negative translation ${ }^{2}$ of $A$. Then the following rule holds:

$$
\left\{\begin{array}{l}
G_{n} A^{\omega}+A C-q f+\Delta \vdash A^{H} \wedge M o n(A) \Rightarrow \\
G_{n} A^{\omega}+\tilde{\Delta} \vdash A \text { and by monotone functional interpretation } \\
\text { one can extract a tuple } \underline{\Psi} \in G_{n} R^{\omega} \text { such that } \\
G_{n} A_{i}^{\omega}+\tilde{\Delta} \vdash \underline{\Psi} \text { satisfies the monotone functional interpretation of } A^{\prime},
\end{array}\right.
$$

where $\tilde{\Delta}:=\left\{\exists Y \leq_{\rho \delta} s \forall x^{\delta}, z^{\eta} G_{0}(x, Y x, z): \forall x^{\delta} \exists y \leq_{\rho} s x \forall z^{\eta} G_{0}(x, y, z) \in \Delta\right\}$. (In particular the second conclusion can be proved in $\left.G_{n} A_{i}^{\omega}+\Delta+b-A C\right)^{3}$.

\footnotetext{
${ }^{2}$ Here we can use Gödel's [5] translation or any of the various negative translations. For a systematical treatment of negative translations see [15].

${ }^{3}$ Here $\mathrm{b}-\mathrm{AC}:=\bigcup_{\delta, \rho \in \mathbf{T}}\left\{\left(\mathrm{b}-\mathrm{AC}^{\delta, \rho}\right)\right\}$ denotes the schema $\left(\mathrm{b}-\mathrm{AC}^{\delta, \rho}\right): \forall Z^{\rho \delta}\left(\forall x^{\delta} \exists y \leq_{\rho} Z x A(x, y, Z) \rightarrow \exists Y \leq_{\rho \delta} Z \forall x A(x, Y x, Z)\right)$.
} 
Remark 29 In theorems 27,28 one may also have tuples ' $\exists \underline{w}$ ' instead of ' $\exists w^{\gamma}$ ' in $A$.

Proof of theorem 27: We assume that

$$
(0) \forall u^{1} \forall v \leq_{\tau} t u \forall h_{1}, \ldots, h_{k}\left(\bigwedge_{i=1}^{k}\left(h_{i} \text { monotone }\right) \rightarrow \exists y_{1}, \ldots, y_{k} \leq_{0} \Psi u \underline{h} \exists w^{\gamma} A_{0}^{H}\right)
$$

(This assumption follows from the implicative premise in the theorem by taking $\Psi u \underline{h}:=$ $\left.\max _{0}\left(\Psi_{1} u \underline{h}, \ldots, \Psi_{k} u \underline{h}\right)\right)$. By $[14]\left(\operatorname{cor} .2 .2 .24\right.$ and rem.2.2.25) one can construct a term $\Psi^{*}[\bar{u}, \underline{h}]^{0}$ such that

1. $\Psi^{*}[u, \underline{h}]$ is built up from $u, \underline{h}, A_{0}, \ldots, A_{n}, S^{1}, 0^{0}, \max _{0}$ only (by application).

2. $\lambda u, \underline{h} \cdot \Psi^{*}[u, \underline{h}] \mathrm{s}-\mathrm{maj} \Psi$ (see [14] for the definition of s-maj).

1) in particular implies:

$\left.1^{*}\right)$ Every occurrence of an $h_{j} \in\left\{h_{1}, \ldots, h_{k}\right\}$ in $\Psi^{*}[u, \underline{h}]$ has the form

$h_{j}\left(r_{n_{1}}, \ldots, r_{n_{j}}\right)$, i.e. $h_{j}$ occurs only with a full stock of arguments but not as a function argument in the form $s\left(h_{j} r_{n_{1}} \ldots r_{n_{l}}\right)$ for some $l<j$.

By 2), $\forall u^{1}\left(u^{M}\right.$ s-maj $u$ ) (where $\left.u^{M} x:=\max _{i \leq x} u i\right)$ and $\left(h_{i}\right.$ monotone $\rightarrow h_{i}$ s-maj $\left.h_{i}\right)$ we have

$\left.2^{*}\right) \mathrm{G}_{n} \mathrm{~A}^{\omega} \vdash \forall u \forall h_{1}, \ldots, h_{k}\left(\bigwedge_{i=1}^{k}\left(h_{i}\right.\right.$ monotone $\left.) \rightarrow \Psi^{*}\left[u^{M}, \underline{h}\right] \geq_{0} \Psi u \underline{h}\right)$.

(Note the the replacement of $h_{i}$ by $h_{i}^{M}:=\lambda x_{1}, \ldots, x_{i} \cdot \max _{\tilde{x}_{1} \leq x_{1}} h\left(\tilde{x}_{1}, \ldots, \tilde{x}_{i}\right)$, which would

$$
\begin{gathered}
\vdots \\
\tilde{x}_{i} \leq x_{i}
\end{gathered}
$$

make the monotonicity assumption on $h_{i}$ superfluous, would destroy property $1^{*}$ ) on which the proof below is based. This is the reason why we have to assume $h_{i}$ to be monotone. In order to overcome this assumption we will use essentially the monotonicity of $A$ ).

Let $r_{1}, \ldots, r_{l}$ be all subterms of $\Psi^{*}\left[u^{M}, \underline{h}\right]$ which occur as an argument of a function $\in\left\{h_{1}, \ldots, h_{k}\right\}$ in $\Psi^{*}\left[u^{M}, \underline{h}\right]$ plus the term $\Psi^{*}\left[u^{M}, \underline{h}\right]$ itself.

Let $\widehat{r}_{j}\left[a_{1}, \ldots, a_{q_{j}}\right]$ be the term which results from $r_{j}$ if every occurrence of a maximal $h_{1}, \ldots, h_{k}$-subterm (i.e. a maximal subterm which has the form $h_{i}\left(s_{1}, \ldots, s_{i}\right)$ for an $i=$ $1, \ldots, k)$ is replaced by a new variable and let $a_{1}, \ldots, a_{q_{j}}$ denote these variables. We now define

$$
\tilde{r}_{j} a_{1} \ldots a_{q_{j}}:=\max \left(\max _{\substack{\tilde{a}_{1} \leq a_{1} \\ \vdots \\ \tilde{a}_{q_{j}} \leq a_{q_{j}}}} \widehat{r}_{j}\left[\tilde{a}_{1}, \ldots, \tilde{a}_{q_{j}}\right], a_{1}, \ldots, a_{q_{j}}\right) .
$$

$\left(\tilde{r}_{j}\right.$ can be defined in $\mathrm{G}_{n} \mathrm{R}^{\omega}$ from $\widehat{r}_{j}$ by successive use of $\left.\Phi_{1}\right)$.

By the construction of $\tilde{r}_{j}$ we get

$$
\mathrm{G}_{n} \mathrm{~A}^{\omega} \vdash\left(\lambda \underline{a} . \tilde{r}_{j} \underline{a} \mathrm{~s}-\mathrm{maj} \lambda \underline{a} . \widehat{r}_{j}\left[a_{1}, \ldots, a_{q_{j}}\right]\right) \wedge \forall \underline{a}\left(\tilde{r}_{j} \underline{a} \geq_{0} a_{1}, \ldots, a_{q_{j}}\right) .
$$


Since $\Psi^{*}\left[u^{M}, \underline{h}\right]$ is built up from $\widehat{r}_{j}, \underline{h}$ only (by substitution) and $\left(h_{i}\right.$ monotone $\rightarrow h_{i}$ s-maj $\left.h_{i}\right), u^{M} \mathrm{~s}-\mathrm{maj} u$, this implies

$$
\mathrm{G}_{n} \mathrm{~A}^{\omega} \vdash \forall u, h_{1}, \ldots, h_{k}\left(\bigwedge_{i=1}^{k}\left(h_{i} \text { monotone }\right) \rightarrow \bar{\Psi}\left[u^{M}, \underline{h}\right] \geq_{0} \Psi^{*}\left[u^{M}, \underline{h}\right] \geq_{0} \Psi u \underline{h}\right),
$$

where $\bar{\Psi}\left[u^{M}, \underline{h}\right]$ is built up as $\Psi^{*}\left[u^{M}, \underline{h}\right]$ but with $\tilde{r}_{j}\left(a_{1}, \ldots, a_{q_{j}}\right)$ instead of $\widehat{r}_{j}\left[a_{1}, \ldots, a_{q_{j}}\right]$.

Summarizing the situation achieved so far we have obtained a term $\bar{\Psi}\left[u^{M}, \underline{h}\right]$ such that

$(\alpha) \forall u^{1} \forall v \leq_{\tau} t u \forall \underline{h}\left(\underline{h}\right.$ monotone $\left.\rightarrow \exists y_{1}, \ldots, y_{k} \leq_{0} \bar{\Psi}\left[u^{M}, \underline{h}\right] \exists w^{\gamma} A_{0}^{H}\right)$.

( $\beta) h_{1}, \ldots, h_{k}$ occur in $\bar{\Psi}\left[u^{M}, \underline{h}\right]$ only as in $\left.1^{*}\right)$, i.e. with all places for arguments filled and not as function arguments themselves.

$(\gamma)$ For $\bar{\Psi}\left[u^{M}, \underline{h}\right]$ and all subterms $s$ which occur as an argument of a function $h_{1}, \ldots, h_{k}$ in $\bar{\Psi}\left[u^{M}, \underline{h}\right]$ we have $\mathrm{G}_{n} \mathrm{~A}^{\omega} \vdash \widehat{s}\left[a_{1}, \ldots, a_{q}\right] \geq_{0} a_{1}, \ldots, a_{q}$, where $\widehat{s}$ results by replacing every occurrence of a maximal $h_{1}, \ldots, h_{k}$-subterm in $s$ by a new variable $a_{l}$.

$(\beta),(\gamma)$ do not depend on any assumption and $(\alpha)$ follows from $(0)$ :

$\mathrm{G}_{n} \mathrm{~A}^{\omega} \vdash(0) \rightarrow(\alpha)$.

In the following we only use $(\alpha)-(\gamma)$ and $\operatorname{Mon}(A)$.

¿From now on let $r_{1}, \ldots, r_{l}$ denote all subterms of $\bar{\Psi}\left[u^{M}, \underline{h}\right]$ which occur as an argument of a function $\in\left\{h_{1}, \ldots, h_{k}\right\}$ in $\bar{\Psi}\left[u^{M}, \underline{h}\right]$ plus $\bar{\Psi}\left[u^{M}, \underline{h}\right]$ itself. $M:=\left\{r_{1}, \ldots, r_{l}\right\}$ (This set formation is meant w.r.t. identity $\equiv$ of terms and not $=_{0}$, i.e. ' $s \in M$ ' means ' $s \equiv r_{1} \vee \ldots \vee s \equiv$ $\left.r_{l}{ }^{\prime}\right)$.

We now show that we can reduce ' $\exists y_{1}, \ldots, y_{k} \leq \bar{\Psi}\left[u^{M}, \underline{h}\right]$ ' in $(\alpha)$ to a disjunction with fixed length, namely to the disjunction over $M$ :

$$
\text { (1) }\left\{\begin{array}{r}
\forall u^{1} \forall v \leq_{\tau} t u \forall \underline{h}\left(\underline{h} \text { monotone on } M \rightarrow \exists s_{1}, \ldots, s_{k} \in M \exists w^{\gamma}\right. \\
\left.A_{0}\left(u, v, s_{1}, h_{1} s_{1}, \ldots, s_{k}, h_{k} s_{1} \ldots s_{k}, w\right)\right) .
\end{array}\right.
$$

Proof of (1): Let $h_{1}, \ldots, h_{k}$ be monotone on $M$. We order the terms $r_{i}$ w.r.t. $\leq_{0}$. The resulting ordered tuple depends of course on $u, h_{1}, \ldots, h_{k}$. For notational simplicity we assume that $r_{1} \leq_{0} \ldots \leq_{0} r_{l}$. We now define (again depending on $u, \underline{h}$ ) functions $\tilde{h}_{1}, \ldots, \tilde{h}_{k}$ by

$$
\begin{gathered}
\tilde{h}_{i} y_{1}^{0} \ldots y_{i}^{0}:=h_{i}\left(r_{j_{y_{1}}}, \ldots, r_{j_{y_{i}}}\right), \text { where } \\
j_{y_{q}}:=\left\{\begin{array}{l}
1, \text { if } y_{q} \leq_{0} r_{1} \\
j+1, \text { if } r_{j}<_{0} y_{q} \leq_{0} r_{j+1} \\
l, \text { if } r_{l}<_{0} y_{q} .
\end{array}\right.
\end{gathered}
$$

Since $l$ (and therefore the number of cases in this definition of $\tilde{h}_{i}$ ) is a (from outside) fixed number depending only on the term structure of $\bar{\Psi}\left[u^{M}, \underline{h}\right]$ but not on $u, \underline{h}$, the functions $\tilde{h}_{i}$ can be defined uniformly in $u, \underline{h}$ within $\mathrm{G}_{n} \mathrm{~A}^{\omega}$. On $M, \tilde{h}_{i}$ equals $h_{i}$.

By the definition of $\tilde{h}_{i}$ and the assumption that $h_{1}, \ldots, h_{k}$ are monotone on $M$ we conclude

$$
\text { (a) } \tilde{h}_{1}, \ldots, \tilde{h}_{k} \text { are monotone everywhere. }
$$


By $(\beta)$ we know that $h_{1}, \ldots, h_{k}$ occur in $\bar{\Psi}\left[u^{M}, \underline{h}\right]$ only in the form $h_{i}\left(s_{1}, \ldots, s_{i}\right)$ for certain terms $s_{1}, \ldots, s_{i} \in M$. Hence we can define the $\underline{h}$-depth of a term $s \in M$ as the maximal number of nested occurrences of $h_{1}, \ldots, h_{k}$ in $s$ and show by induction on this rank (on the meta-level):

$$
\text { (b) }\left\{\begin{array}{l}
\bigwedge_{i=1}^{l}\left(r_{i}={ }_{0} \tilde{r}_{i}\right), \text { where } \tilde{r}_{i} \text { results if in } r_{i} \in M \text { the functions } h_{1}, \ldots, h_{k} \\
\text { are replaced by } \tilde{h}_{1}, \ldots, \tilde{h}_{k} \text { everywhere. } \\
\text { In particular } \bar{\Psi}\left[u^{M}, \underline{\tilde{h}}\right]==_{0} \bar{\Psi}\left[u^{M}, \underline{h}\right] .
\end{array}\right.
$$

By $(\alpha),(a)$ and $(b)$ it follows (for all $u^{1}, v \leq t u$ and all $\underline{h}$ which are monotone on $M$ ) that

$$
\text { (c) } \exists y_{1}, \ldots, y_{k} \leq_{0} \bar{\Psi}\left[u^{M}, \underline{h}\right] \exists w^{\gamma} A_{0}\left(u, v, y_{1}, \tilde{h}_{1} y_{1}, \ldots, y_{k}, \tilde{h}_{k} y_{1} \ldots y_{k}, w\right) .
$$

Let $y_{1}, \ldots, y_{k} \leq_{0} \bar{\Psi}\left[u^{M}, \underline{h}\right]$ be such that $(c)$ is fulfilled. Because of $\tilde{h}_{i} y_{1} \ldots y_{i}=h_{i}\left(r_{j_{y_{1}}}, \ldots, r_{j_{y_{i}}}\right)$ this implies

$$
\text { (d) } \exists w^{\gamma} A_{0}\left(u, v, y_{1}, h_{1} r_{j_{y_{1}}}, \ldots, y_{k}, h_{k} r_{j_{y_{1}}} \ldots r_{j_{y_{k}}}, w\right) .
$$

With $y_{q} \leq r_{j_{y_{q}}}$ for $q=1, \ldots k$ (since $y_{q} \leq \bar{\Psi}\left[u^{M}, \underline{h}\right] \leq r_{l}$-because of $\bar{\Psi}\left[u^{M}, \underline{h}\right] \in M$ and the $y_{q}$-assumption- the case ' $y_{q}>r_{l}$ ' does not occur) and $\operatorname{Mon}(A)$ we conclude

$$
\exists w^{\gamma} A_{0}\left(u, v, r_{j_{y_{1}}}, h_{1} r_{j_{y_{1}}}, \ldots, r_{j_{y_{k}}}, h_{k} r_{j_{y_{1}}} \ldots r_{j_{y_{k}}}, w\right)
$$

and therefore

$$
\text { (e) } \exists s_{1}, \ldots, s_{k} \in M \exists w^{\gamma} A_{0}\left(u, v, s_{1}, h_{1} s_{1}, \ldots, s_{k}, h_{k} s_{1} \ldots s_{k}, w\right) .
$$

This concludes the proof of (1) which can easily be carried out in $\mathrm{G}_{n} \mathrm{~A}^{\omega}$ (assuming $\operatorname{Mon}(A),(\alpha)$ and using $(\beta))$, i.e.

$$
\mathrm{G}_{n} \mathrm{~A}^{\omega} \vdash \operatorname{Mon}(A) \wedge(\alpha) \rightarrow(1) .
$$

We now define $N:=\bigcup_{i=1}^{k} N_{i}$, where $N_{i}:=\left\{h_{i}\left(s_{1}, \ldots, s_{i}\right): s_{1}, \ldots, s_{i} \in M\right\}$ (Again this set is meant w.r.t. identity $\equiv$ between terms). With the terms in $N$ we associate new number variables according to their $\underline{h}$-depth as follows: Let $p$ the maximal $\underline{h}$-depth of all terms $\in N$.

1. Let $t \in N$ be a term with $\underline{h}-\operatorname{depth}(t)=p$. Then $t \mapsto y_{i}^{1}$, if $t \in N_{i}$.

2. Let $t \in N$ be a term with $\underline{h}-\operatorname{depth}(t)=p-1$. Then $t \mapsto y_{i}^{2}$, if $t \in N_{i}$.

p. Let $t \in N$ be a term with $\underline{h}-\operatorname{depth}(t)=1$. Then $t \mapsto y_{i}^{p}$, if $t \in N_{i}$.

This association of variables to the terms in $N$ has the following properties:

(i) Terms $s_{1}, s_{2} \in N$ with different $\underline{h}$-depth have different variables associated with.

(ii) If $s_{1}, s_{2} \in N$ have the same $\underline{h}$-depth, then the variables associated with $s_{1}$ and $s_{2}$ are equal iff $s_{1}, s_{2} \in N_{i}$ for an $i=1, \ldots, k$. 
For $r \in M \cup N$ we define $\widehat{r}$ as the term which results if every maximal $\underline{h}$-subterm occurring in $r$ is replaced by its associated variable. Thus $\widehat{r}$ does not contain $h_{1}, \ldots, h_{k}$. For $r \in N, \widehat{r}$ is just the variable associated with $r . \widehat{M}:=\{\widehat{r}: r \in M\}$.

We now show that (1) implies a certain index function-free (i.e. $h_{1}, \ldots, h_{k}$-free) disjunction ((2) below):

For $q$ with $2 \leq q \leq p$ let $\widehat{r}_{1}^{q}, \ldots, \widehat{r}_{n_{q}}^{q}$ be all terms $\in \widehat{M}$ whose smallest upper index $i$ of a variable $y_{j}^{i}$ occurring in them equals $q$ (i.e. there occurs a variable $y_{j}^{q}$ in the term and for all variables $y_{m}^{i}$ occurring in the term, $i \geq q$ holds). Since for $r \in M$ the $\underline{h}$-depth of $h_{1}(r) \in N$ is strictly greater than those of subterms of $r$, there are no terms $\widehat{r} \in \widehat{\widehat{M}}$ containing a variable $y_{j}^{1} . \widehat{r}_{1}^{p+1}, \ldots, \widehat{r}_{n_{p+1}}^{p+1}$ denote those terms $\in \widehat{M}$ which do not contain any variable $y_{j}^{i}$ at all.

We now show that (1) implies (for all $u$ and for all $v \leq t u$ )

$$
\text { (2) }\left\{\begin{aligned}
\forall y_{1}^{1}, \ldots, y_{k}^{1} ; \ldots ; y_{1}^{p}, \ldots, y_{k}^{p} \\
\left((+) \rightarrow \underset{\widehat{s}_{1}, \ldots, \widehat{s}_{k} \in \widehat{M}}{\bigvee} \exists w^{\gamma} A_{0}\left(u, v, \widehat{s}_{1}, \widehat{h_{1} s_{1}}, \ldots, \widehat{s}_{k}, h_{k} \widehat{s_{1} \ldots} s_{k}, w\right)\right),
\end{aligned}\right.
$$

where $^{4}$

$$
(+): \equiv\left\{\begin{array}{l}
\bigwedge_{\substack{q=1, \ldots, p-1 \\
l=1, \ldots, p-q}}^{\wedge}\left(y_{1}^{q}, \ldots, y_{k}^{q}>\widehat{r}_{1}^{q+l}, \ldots, \widehat{r}_{n_{q+l}}^{q+l}, y_{1}^{q+l}, \ldots, y_{k}^{q+l}\right) \wedge \\
\bigwedge_{q=1, \ldots, p}\left(y_{1}^{q}, \ldots, y_{k}^{q}>\widehat{r}_{1}^{p+1}, \ldots, \widehat{r}_{n_{p+1}}^{p+1}\right) .
\end{array}\right.
$$

Assume that there are values $y_{1}^{1}, \ldots, y_{k}^{1} ; \ldots ; y_{1}^{p}, \ldots, y_{k}^{p}$ such that $(+)$ holds and

$$
\bigwedge_{\widehat{s}_{1}, \ldots, \widehat{s}_{k} \in \widehat{M}} \neg \exists w^{\gamma} A_{0}\left(u, v, \widehat{s}_{1}, \widehat{h_{1} s_{1}}, \ldots, \widehat{s}_{k}, h_{k} \widehat{s_{1} \ldots} s_{k}, w\right) .
$$

We construct (working in $\mathrm{G}_{n} \mathrm{~A}^{\omega}$ ) functions $h_{1}, \ldots, h_{k}$ which are monotone on $M$ and satisfy

$$
\forall s_{1}, \ldots, s_{k} \in M \neg \exists w A_{0}\left(u, v, s_{1}, h_{1} s_{1}, \ldots, s_{k}, h_{k} s_{1} \ldots s_{k}, w\right)
$$

yielding a contradiction to (1): Define for $i=1, \ldots, k$

$$
\begin{aligned}
& h_{i}\left(x_{1}, \ldots, x_{i}\right):= \\
& \left\{\begin{array}{l}
\min _{i} \operatorname{mil}_{1 \leq i}\left(q_{l}\right)-1 \\
0^{0}, \text { otherwise. }^{5}
\end{array} \text { if } \exists \widehat{r}_{j_{1}}^{q_{1}}, \ldots, \widehat{r}_{j_{i}}^{q_{i}} \in \widehat{M}\left(\left(x_{1}, \ldots, x_{i}\right)={ }_{0}\left(\widehat{r}_{j_{1}}^{q_{1}}, \ldots, \widehat{r}_{j_{i}}^{q_{i}}\right)\right)\right.
\end{aligned}
$$

\footnotetext{
${ }^{4}$ Here $a_{1}, \ldots, a_{k}>b_{1}, \ldots, b_{l}$ means $\bigwedge_{\substack{1 \leq i \leq k \\ 1 \leq j \leq l}}\left(a_{i}>b_{j}\right)$.

In (2) above we actually show the disjunction ' $\bigvee_{s_{1}, \ldots, s_{k} \in M} \exists w^{\gamma} A_{0}\left(u, v, \widehat{s_{1}}, \widehat{h_{1} s_{1}}, \ldots\right)$ ' instead of ' ${\widehat{s_{1}}, \ldots, \widehat{s}_{k} \in \widehat{M}} \exists w^{\gamma} A_{0}\left(u, v, \widehat{s}_{1}, \widehat{h_{1} s_{1}}, \ldots\right)$ '. However the later follows from the former disjunction by contraction since $\widehat{s}_{1} \equiv \widehat{s}_{1}^{\prime} \wedge \ldots \wedge \widehat{s}_{i} \equiv \widehat{s}_{i}^{\prime}$ implies $h_{i} \widehat{s_{1} \ldots} s_{i} \equiv h_{i} \widehat{s_{1}^{\prime} \ldots} s_{i}^{\prime}$ for $s_{1}, s_{1}^{\prime}, \ldots, s_{i}^{\prime}, s_{i}^{\prime} \in M$. Alternatively we could also use the non-contracted disjunction in the following proof.
} 
We have to show:

(i) The $h_{i}$ are well-defined functions : $\underbrace{\mathbb{N} \times \ldots \times \mathbb{N}}_{i} \rightarrow \mathbb{N}$ and the definition above can be carried out in $\mathrm{G}_{n} \mathrm{~A}^{\omega}$.

(ii) $\widehat{r}={ }_{0} r$ for all $r \in M \cup N$ (for these $h_{1}, \ldots, h_{k}$ ).

(iii) $h_{1}, \ldots, h_{k}$ are monotone on $\widehat{M}$ (and hence -by (ii)- on $M$ ).

Ad (i): Consider $\left(\widehat{r}_{j_{1}}^{q_{1}}, \ldots, \widehat{r}_{j_{i}}^{q_{i}}\right)$ and $\left(\widehat{r}_{\tilde{j}_{1}}^{\tilde{q}_{1}}, \ldots, \widehat{r}_{\tilde{j}_{i}}^{\tilde{q}_{i}}\right)$. We show that $y_{i}^{\min _{1 \leq l \leq i}\left(q_{l}\right)-1} \neq y_{i}^{\min _{1 \leq l \leq i}\left(\tilde{q}_{l}\right)-1}$ implies $\left(\widehat{r}_{j_{1}}^{q_{1}}, \ldots, \widehat{r}_{j_{i}}^{q_{i}}\right) \neq\left(\widehat{r}_{\tilde{j}_{1}}^{\tilde{q}_{1}}, \ldots, \widehat{r}_{\tilde{j}_{i}}^{\tilde{q}_{i}}\right)$ :

We may assume $\min _{1 \leq l \leq i}\left(q_{l}\right)<\min _{1 \leq l \leq i}\left(\tilde{q}_{l}\right)$. Let $l_{0}$ be such that $q_{l_{0}}=\min _{1 \leq l \leq i}\left(q_{l}\right) \wedge 1 \leq l_{0} \leq i . \widehat{r}_{j_{l_{0}}}^{q_{l_{0}}}$ contains a variable $y_{d}^{q_{l_{0}}}$ for some $d=1, \ldots, k$. By the property $(\gamma)$ of $\bar{\Psi}\left[u^{M}, \underline{h}\right]$ this implies

$$
\widehat{r}_{j_{0}}^{q_{l_{0}}} \geq y_{d}^{q_{l_{0}}} \stackrel{(+), q_{l_{0}}<\tilde{q}_{l_{0}}}{>} \widehat{r}_{\tilde{j}_{l_{0}}}^{\tilde{q}_{l_{0}}} \text { and thus }\left(\widehat{r}_{j_{1}}^{q_{1}}, \ldots, \widehat{r}_{j_{i}}^{q_{i}}\right) \neq\left(\widehat{r}_{\tilde{j}_{1}}^{\tilde{q}_{1}}, \ldots, \widehat{r}_{\tilde{j}_{i}}^{\tilde{q}_{i}}\right) .
$$

Hence $h_{i}$ can be defined in $\mathrm{G}_{n} \mathrm{~A}^{\omega}$ by a definition by cases which are pairwise exclusive.

Ad (ii): (ii) follows from the definition of $h_{1}, \ldots, h_{k}$ by induction on the $\underline{h}$-depth of $r$.

Ad (iii): Assume $\bigwedge_{l=1}^{i}\left(\widehat{r}_{j_{l}}^{q_{l}} \leq_{0} \widehat{r}_{\tilde{j}_{l}}^{\tilde{q}_{l}}\right)$. Let $l_{0}\left(1 \leq l_{0} \leq i\right)$ be such that $q_{l_{0}}=\min _{1 \leq l \leq i}\left(q_{l}\right)$. By contraposition of the implication established in the proof of (i) one has: $\min _{1 \leq l \leq i}\left(q_{l}\right) \geq \min _{1 \leq l \leq i}\left(\tilde{q}_{l}\right)$.

Case 1: $\min _{1 \leq l \leq i}\left(q_{l}\right)=\min _{1 \leq l \leq i}\left(\tilde{q}_{l}\right)$. Then (by $h_{i}$-definition)

$$
h_{i}\left(\widehat{r}_{j_{1}}^{q_{1}}, \ldots, \widehat{r}_{j_{i}}^{q_{i}}\right)=y_{i}^{\min \left(q_{l}\right)-1}=y_{i}^{\min \left(\tilde{q}_{l}\right)-1}=h_{i}\left(\widehat{r}_{\tilde{j}_{1}}^{\tilde{q}_{1}}, \ldots, \widehat{r}_{\tilde{j}_{i}}^{\tilde{q}_{i}}\right) .
$$

Case 2: $q_{l_{0}}=\min _{1 \leq l \leq i}\left(q_{l}\right)>\min _{1 \leq l \leq i}\left(\tilde{q}_{l}\right)=\tilde{q}_{\tilde{l}_{0}}\left(\right.$ where $\left.1 \leq l_{0}, \tilde{l}_{0} \leq i\right)$. Then

$$
h_{i}\left(\widehat{r}_{j_{1}}^{q_{1}}, \ldots, \widehat{r}_{j_{i}}^{q_{i}}\right)=y_{i}^{q_{l_{0}}-1} \stackrel{(+)}{<} y_{i}^{\tilde{q}_{\tilde{l}_{0}}-1}=h_{i}\left(\widehat{r}_{\tilde{j}_{1}}^{\tilde{q}_{1}}, \ldots, \widehat{r}_{\tilde{j}_{i}}^{\tilde{q}_{i}}\right) .
$$

Hence $h_{1}, \ldots, h_{k}$ are monotone on $\widehat{M}$ and therefore (by (ii)) on $M$, which concludes the proof of (2) from (1) in $\mathrm{G}_{n} \mathrm{~A}^{\omega}$ (using $(\beta),(\gamma)$ ). Since (1) follows (in $\mathrm{G}_{n} \mathrm{~A}^{\omega}$ ) from $\operatorname{Mon}(A) \wedge(\alpha)$ (using $(\beta)$ ), and

$$
F: \equiv \forall u^{1} \forall v \leq_{\tau} t u \forall \underline{h}\left(\underline{h} \text { monotone } \rightarrow \exists y_{1}, \ldots, y_{k} \leq_{0} \Psi u \underline{h} \exists w^{\gamma} A_{0}^{H}\right)
$$

implies (in $\left.\mathrm{G}_{n} \mathrm{~A}^{\omega}\right)(\alpha)$, we have shown altogether

$$
\text { (3) }\left\{\begin{array}{l}
\mathrm{G}_{n} \mathrm{~A}^{\omega}+\operatorname{Mon}(A) \vdash \\
F \rightarrow\left[v \leq t u \wedge(+) \underset{\widehat{s}_{1}, \ldots, \widehat{s}_{k} \in \widehat{M}}{\bigvee} \exists w^{\gamma} A_{0}\left(u, v, \widehat{s}_{1}, \widehat{h_{1} s_{1}}, \ldots, \widehat{s_{k}}, \widehat{h_{k}} \widehat{s_{1} \ldots s_{k}}, w\right)\right] .
\end{array}\right.
$$

${ }^{5}$ For $\widehat{r}_{j_{i}}^{q_{i}} \in \widehat{M}$ we have $q_{i} \geq 2$ since e.g. $h_{1} r_{j_{i}}(\in N)$ has an $\underline{h}$-depth which is strictly greater than those of subterms in $r_{j_{i}}$. 
It remains to show that (3) implies

(4) $\mathrm{G}_{n} \mathrm{~A}^{\omega}+\operatorname{Mon}(A) \vdash F \rightarrow A$.

We prove this by a suitable application of quantifier introduction rules: We start with the variables with smallest upper index, i.e. $y_{1}^{1}, \ldots, y_{k}^{1}$. Under these variables we first take those of maximal lower index, i.e. with $y_{k}^{1}$ : We split the assumption

$$
(+) \equiv\left\{\begin{array}{l}
\bigwedge_{\substack{q=1, \ldots, p-1 \\
l=1, \ldots, p-q}}\left(y_{1}^{q}, \ldots, y_{k}^{q}>\widehat{r}_{1}^{q+l}, \ldots, \widehat{r}_{n_{q+l}}^{q+l}, y_{1}^{q+l}, \ldots, y_{k}^{q+l}\right) \wedge \\
\bigwedge_{q=1, \ldots, p}\left(y_{1}^{q}, \ldots, y_{k}^{q}>\widehat{r}_{1}^{p+1}, \ldots, \widehat{r}_{n_{p+1}}^{p+1}\right)
\end{array}\right.
$$

as well as the disjunction

$$
A^{d}: \equiv \bigvee_{\widehat{s}_{1}, \ldots, \widehat{s}_{k} \in \widehat{M}} \exists w^{\gamma} A_{0}\left(u, v, \widehat{s}_{1}, \widehat{h_{1} s_{1}}, \ldots, \widehat{s}_{k}, h_{k} \widehat{s_{1} \ldots} s_{k}, w\right)
$$

into the part in which $y_{k}^{1}$ occurs and into its $y_{k}^{1}$-free part:

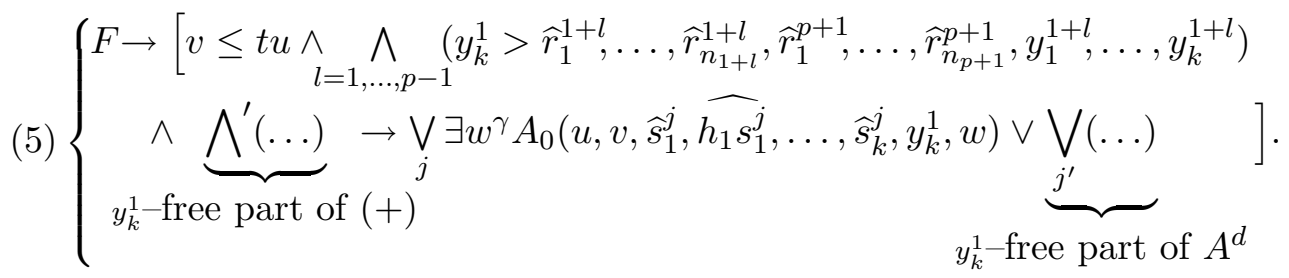

$y_{k}^{1}$ does not occur at any place other than indicated. Hence $\forall$-introduction applied to $y_{k}^{1}$ yields:

$$
\text { (6) } F \rightarrow \forall y_{k}^{1}\left[v \leq t u \wedge \bigwedge_{l}\left(y_{k}^{1}>\ldots\right) \wedge \bigwedge^{\prime}(\ldots) \rightarrow \bigvee_{j} \exists w^{\gamma} A_{0}\left(\ldots, y_{k}^{1}, w\right) \vee \bigvee_{j^{\prime}}(\ldots)\right]
$$

where $y_{k}^{1}$ does not occur at any place other than indicated.

Using $\operatorname{Mon}(A)$ this implies

$$
\text { (7) } F \rightarrow\left[v \leq t u \wedge \bigwedge^{\prime}(\ldots) \rightarrow \forall y_{k}^{1} \bigvee_{j} \exists w^{\gamma} A_{0}\left(\ldots, y_{k}^{1}, w\right) \vee \bigvee_{j^{\prime}}(\ldots)\right]
$$

(Proof: In (6) put

$$
\tilde{y}_{k}^{1}:=\max _{1 \leq l \leq p-1} \max \left(y_{k}^{1}, \widehat{r}_{1}^{1+l}, \ldots \widehat{r}_{n_{1+l}}^{1+l}, \widehat{r}_{1}^{p+1}, \ldots, \widehat{r}_{n_{p+1}}^{p+1}, y_{1}^{1+l}, \ldots, y_{k}^{1+l}\right)+1 \text { for } y_{k}^{1} \text {. }
$$

(6) then gives

$$
F \rightarrow\left[v \leq t u \wedge \bigwedge^{\prime}(\ldots) \rightarrow \bigvee_{j} \exists w^{\gamma} A_{0}\left(\ldots, \tilde{y}_{k}^{1}, w\right) \vee \bigvee_{j^{\prime}}(\ldots)\right]
$$


$\operatorname{Mon}(A)$ and $\underset{j}{\bigvee} \exists w^{\gamma} A_{0}\left(\ldots, \tilde{y}_{k}^{1}, w\right)$ imply $\bigvee_{j} \exists w^{\gamma} A_{0}\left(\ldots, y_{k}^{1}, w\right)$, since $\tilde{y}_{k}^{1} \geq y_{k}^{1}$. Now $\forall$-introduction applied to $y_{k}^{1}$ and shifting $\forall y_{k}^{1}$ in front of $\underset{j}{\bigvee}$, which is possible since $y_{k}^{1}$ occurs only in this disjunction, proves (7)).

Again by $\operatorname{Mon}(A)$ we obtain

$$
\bigvee_{j} \forall y_{k}^{1} \exists w^{\gamma} A_{0}\left(\ldots, y_{k}^{1}, w\right)
$$

from $\forall y_{k}^{1} \bigvee_{j} \exists w^{\gamma} A_{0}\left(\ldots, y_{k}^{1}, w\right)$ :

Assume $\bigwedge \exists y_{k}^{1} \forall w^{\gamma} \neg A_{0}\left(\ldots, y_{k}^{1}, w\right)$. Then $\exists y \wedge \exists y_{k}^{1} \leq_{0} y \forall w^{\gamma} \neg A_{0}\left(\ldots, y_{k}^{1}, w\right)$. Using $\operatorname{Mon}(A)$ this implies $\exists y \bigwedge_{j} \forall w^{\gamma} \neg A_{0}(\ldots, y, w)$.

Hence (7) implies (since $y_{k}^{1}$ does not occur in $\widehat{s}_{k}^{j}$ )

$$
\text { (8) }\left\{\begin{aligned}
& F \rightarrow {\left[v \leq t u \wedge \wedge^{\prime}(\ldots) \rightarrow\right.} \\
&\left.\bigvee_{j} \exists x \forall y \exists w A_{0}\left(u, v, \widehat{s}_{1}^{j}, \widehat{h_{1} s_{1}^{j}}, \ldots, h_{k-1} \widehat{s_{1}^{j} \ldots} s_{k-1}^{j}, x, y, w\right) \vee \underset{j^{\prime}}{\bigvee}(\ldots)\right] .
\end{aligned}\right.
$$

Next we apply the same procedure to the variable $y_{k-1}^{1}$ and then to $y_{k-2}^{1}$ and so on until all $y_{1}^{1}, \ldots, y_{k}^{1}$ are bounded. We then continue with $y_{k}^{2}, y_{k-1}^{2}$ and so on. This corresponds to the sequence of quantifications used in the usual proofs of Herbrand's theorem in order to show that there is a direct proof from the Herbrand disjunction of a first-order formula to this formula itself: By taking always variables of minimal upper index it is ensured that any variable to which the $\forall$-introduction rule is applied occurs in the disjunction $\bigvee A_{0}$ only at places where it is universal quantified in the original formula $A$. By quantifying under these variables firstly the one with maximal lower index one ensures that a universal quantifier is introduced only if the quantifiers which stand behind this one in $A$ have already been introduced. In addition to these two reasons for the special sequence of quantifications there is in our situation another (essentially used) property which is fulfilled only if variables which have minimal upper index are quantified first: If $y_{j}^{i}$ has minimal index $i$ (under all variables which still have to be quantified), then $y_{j}^{i}$ occurs in the still remaining part of the implicative assumption $(+)$ only in the form ' $y_{j}^{i}>\left(\ldots y_{j}^{i}\right.$-free...)'. So we are in the situation at the begining for $y_{k}^{1}$ and are able to eliminate this part of $(+)$ which is connected with $y_{j}^{i}$ altogether using $\operatorname{Mon}(A)$ (as we have shown for $y_{k}^{1}$ ).

Finally we have derived

$$
\text { (9) } F \rightarrow\left[v \leq t u \rightarrow \bigvee \exists x_{1}^{0} \forall y_{1}^{0} \ldots \exists x_{k}^{0} \forall y_{k}^{0} \exists w^{\gamma} A_{0}\left(u, v, x_{1}, y_{1}, \ldots, x_{k}, y_{k}, w\right)\right.
$$

and therefore (by contraction of $\bigvee$ )

$$
\text { (10) } F \rightarrow\left[v \leq t u \rightarrow \exists x_{1}^{0} \forall y_{1}^{0} \ldots \exists x_{k}^{0} \forall y_{k}^{0} \exists w^{\gamma} A_{0}\left(u, v, x_{1}, y_{1}, \ldots, x_{k}, y_{k}, w\right)\right.
$$


which (by $\forall$-introduction applied to $u, v$ ) yields

$$
\text { (11) } F \rightarrow A \text {. }
$$

Remark 210 The proof of thm.27 also works for various other systems $\mathcal{T}$ and domains of terms $S$ than $\mathrm{G}_{n} \mathrm{~A}^{\omega}$ and $\mathrm{G}_{n} \mathrm{R}^{\omega}$. What actually is used in the proof is:

1. Every term $\Psi^{\rho} \in S$ with $\operatorname{deg}(\rho) \leq 2$ has a majorant $\Psi^{*}\left[\underline{h}^{1}\right]$ such that

(i) $\mathcal{T} \vdash \lambda \underline{h} \cdot \Psi^{*}[\underline{h}] \mathrm{s}-$ maj $\Psi$,

(ii) $\Psi^{*}[\underline{h}]$ is built up only from $\underline{h}$ and terms $\in S$ of type level $\leq 1$ (by substitution).

2. $S$ is (provably in $\mathcal{T}$ ) closed under the successor, definition by cases, $\lambda$-abstraction and contains the variable maximum-functional $\Phi_{1}$.

Condition 1) is a sort of an upper bound for the complexity of $\mathcal{T}, S$. E.g. 1) is not satisfied if $S$ contains the iteration functional $\Phi_{i t}$ defined by $\Phi_{i t} 0 y f={ }_{0} y, \Phi_{i t} x^{\prime} y f={ }_{0} f\left(\Phi_{i t} x y f\right)$ (Note that $\Phi_{i t}$ is primitive recursive in the usual sense of [6] and not only in the extended sense of [5]). In the next paragraph we will show that thm.27 becomes false if $\mathrm{G}_{n} \mathrm{R}^{\omega}$ is replaced by $\widehat{P R}^{\omega}$ (see also remark 214 ). Since $\Phi_{i t}$ is on some sense the simplest functional for which 1) fails, this shows that the upper bound provided by 1) is quite sharp. 1) essentially says that $\Psi^{001}$ can be majorized by a term $\Psi^{*}\left[x^{0}, h^{1}\right]$ which uses $h$ only at a fixed number of arguments, i.e. there exists a fixed number $n$ (which depends only on the structure of $\Psi^{*}$ but not on $x, h)$ such that for all $h, x$ the value of of $\Psi^{*}[x, h]$ only depends on (at most) $n$-many $h$-values. Let us illustrate this by an example: $\Phi$ defined by $\Phi h x=\max (h 0, \ldots, h x)$ depends on $x+1$-many $h$-values but is majorized by $\Phi^{*}$ defined by $\Phi^{*} h x:=h x$ which for every $x$ depends only on one $h$-value, namely on $h x$. If a term $\Psi$ has a majorant which satisfies 1 ) we say that $\Psi$ is majorizable with finite support. One easily convinces oneself that $\Phi_{i t}$ is not majorizable with finite support.

2 ) is a lower bound on the complexity of $\mathcal{T}, S$, which also is essential. E.g. take $\mathcal{T}:=\mathcal{L}^{2}$ and $S:=\left\{0^{0}\right\}$, where $\mathcal{L}^{2}$ is first-order logic with $={ }_{0}, \leq_{0}$ extended by quantification over functions and two constants $0^{0}, 1^{0}$. Consider now

$$
G: \equiv \exists x^{0} \forall y^{0} \exists z^{0}, f^{1}\left(F_{0}(f, z) \rightarrow A_{0}(x, y)\right),
$$

where $F_{0}(f, z): \equiv(f z=0 \wedge 0 \neq 1)$ and $A_{0}(x, y): \equiv(y \neq 0 \wedge x=x \rightarrow \perp)$. Then

$$
\mathcal{L}^{2} \vdash \forall g^{1} \exists x, z \leq_{0} 0 \exists f\left(F_{0}(f, z) \rightarrow A_{0}(x, g x)\right) \wedge \operatorname{Mon}(G), \text { but } \mathcal{L}^{2} \forall G,
$$

i.e. thm.27 fails for $\mathcal{L}^{2}, S$. If however $\mathcal{L}^{2}$ is extended by $\lambda$-abstraction, then $G$ becomes derivable since we can form $f:=\lambda x^{0} \cdot 1^{0}$ now.

Let $F^{-}$denote the 'non-standard' axiom introduced in [14] (def.4.16) and $\mathrm{WKL}_{\text {seq }}^{2}$ be the generalization of the binary König's lemmas WKL as defined in [14](def.4.25). Theorem 27 combined with the elimination procedure for $F^{-}$from [14] yields the following new conservation result for $\mathrm{WKL}_{\text {seq }}^{2}$ : 
Corollary 211 Let $A$ be as in def.26 and thm.27, $n \geq 3$. Then ${ }^{6}$

1. $G_{n} A^{\omega} \oplus F^{-} \oplus A C-q f \vdash A^{H} \Rightarrow G_{n} A^{\omega}+\operatorname{Mon}(A) \vdash A$. In particular: $G_{n} A^{\omega} \oplus F^{-} \oplus A C-q f \vdash A \Rightarrow G_{n} A^{\omega}+\operatorname{Mon}(A) \vdash A$.

2. $G_{n} A^{\omega} \oplus W K L_{\text {seq }}^{2} \oplus A C-q f \vdash A^{H} \Rightarrow G_{n} A^{\omega}+M o n(A) \vdash A$.

In particular:

$G_{n} A^{\omega} \oplus W K L_{\text {seq }}^{2} \oplus A C-q f \vdash A \Rightarrow G_{n} A^{\omega}+\operatorname{Mon}(A) \vdash A$.

If $\tau \leq 1$ (in $A$ ) then $G_{n} A^{\omega} \oplus F^{-} \oplus A C-q f$ can be replaced by

$E-G_{n} A^{\omega}+F^{-}+A C^{\alpha, \beta}-q f$ (with $(\alpha=0 \wedge \beta \leq 1)$ or $(\alpha=1 \wedge \beta=0)$ ).

An analogous result holds for the corresponding variant of $G_{n} A^{\omega}$ where the universal axioms 9) are replaced by the schema of quantifier-free induction.

Proof: 1) By [14](thm.4.21 and remark 3.2.4) $\mathrm{G}_{n} \mathrm{~A}^{\omega} \oplus F^{-} \oplus \mathrm{AC}-\mathrm{qf} \quad \vdash A^{H}$ implies the extractability of a $\Psi \in \mathrm{G}_{n} \mathrm{R}^{\omega}$ such that

$$
\mathrm{G}_{n} \mathrm{~A}^{\omega} \vdash \forall u^{1} \forall v \leq_{\tau} t u \forall \underline{h} \exists y_{1}, \ldots, y_{k} \leq_{0} \Psi u \underline{h} \exists w^{\gamma} A_{0}^{H} .
$$

Theorem 27 now yields $\mathrm{G}_{n} \mathrm{~A}^{\omega}+\operatorname{Mon}(A) \vdash A$.

2) follows from 1) by [14](cor.4.28).

Remark 212 Cor.211 is optimal in the following sense: For simplicity let us consider only the variant of $G_{3} A^{\omega}$ with the universal axioms replaced by the schema of quantifier-free induction and let us denote this system for the moment also by $G_{3} A^{\omega}$. Then $G_{3} A^{\omega} \oplus W K L_{\text {seq }}^{2} \oplus A C^{0,0_{-}}$ qf is neither conservative over $G_{3} A^{\omega}$ w.r.t. sentences $\forall u^{2} \exists w^{1} A_{0}(u, w)$ nor w.r.t. $\Pi_{3}^{0}$-sentences, which do not satisfy Mon $(A)$. The first assertion follows analogously to the proof of 4.11 (ii) in [8]. The second claim follows from the fact that even $G_{3} A^{\omega} \oplus A C^{0,0}$-qf proves the $\Sigma_{1}^{0}$ collection principle, whereas $G_{3} A^{\omega}$ - which is conservative over its first-order fragment, i.e. over the Kalmar-elementary arithmetic - does not prove this principle (see the proof of thm.1 in [17]). Since every instance of the $\Sigma_{1}^{0}$-collection principle (having only arithmetical parameters) can be prenexed into a $\Pi_{3}^{0}$-sentence, already $G_{3} A^{\omega} \oplus A C^{0,0}-q f$ is not $\Pi_{3}^{0}$-conservative over $G_{3} A^{\omega}$. The condition Mon $(A)$ in cor.211 just rules out any non-trivial instances of collection.

\section{Proof of theorem 28:}

$\mathrm{G}_{n} \mathrm{~A}^{\omega}+\Delta+\mathrm{AC}-\mathrm{qf} \vdash A^{H}$ implies the extractability by monotone functional interpretation (see [14] (thm.3.2.2, rem.3.2.4 and the remarks after 3.2.6)) of uniform bounds $\Psi_{1}, \ldots, \Psi_{k} \in$ $\mathrm{G}_{n} \mathrm{R}^{\omega}$ on $\exists y_{i}$ (provably in $\mathrm{G}_{n} \mathrm{~A}_{i}^{\omega}+\tilde{\Delta}$, where

\footnotetext{
${ }^{6}$ Here $\oplus$ means that $F^{-}$and $\mathrm{AC}-\mathrm{qf}$ must not be used in the proof of the premise of an application of the quantifier-free rule of extensionality QF-ER. $\mathrm{G}_{n} \mathrm{~A}^{\omega}$ satisfies the deduction theorem w.r.t $\oplus$ but not w.r.t + . In fact the theorem also holds for $\left(\mathrm{G}_{n} \mathrm{~A}^{\omega}+\mathrm{AC}-\mathrm{qf}\right) \oplus F^{-}$since the deduction property is used in the proof only for $F^{-}$.
} 
$\left.\tilde{\Delta}:=\left\{\exists Y \leq_{\rho \delta} s \forall x^{\delta}, z^{\eta} G_{0}(x, Y x, z): \forall x^{\delta} \exists y \leq_{\rho} s x \forall z^{\eta} G_{0}(x, y, z) \in \Delta\right\}\right)$ which depend only on $u$ and $\underline{h}$ :

(1) $\mathrm{G}_{n} \mathrm{~A}_{i}^{\omega}+\tilde{\Delta} \vdash \forall u \forall v \leq t u \forall \underline{h} \exists y_{1} \leq_{0} \Psi_{1} u \underline{h} \ldots \exists y_{k} \leq_{0} \Psi_{k} u \underline{h} \exists w A_{0}^{H}$.

The assumption $\mathrm{G}_{n} \mathrm{~A}^{\omega}+\Delta+\mathrm{AC}-\mathrm{qf} \vdash \operatorname{Mon}(A)$ implies (by monotone functional interpretation, since $\operatorname{Mon}(A)$ is implied by the monotone functional interpretation of its negative translation) that

$$
\text { (2) } \mathrm{G}_{n} \mathrm{~A}_{i}^{\omega}+\tilde{\Delta} \vdash \operatorname{Mon}(A) \text {. }
$$

Theorem 27 combined with (1) and (2) yields

$$
\mathrm{G}_{n} \mathrm{~A}^{\omega}+\tilde{\Delta} \vdash A \text {. }
$$

The second part of the theorem now follows by monotone functional interpretation, since $\tilde{\Delta}$ also is a set of allowed axioms $\Delta$ in $[14]\left(\right.$ thm.3.2.2) and $\mathrm{G}_{n} \mathrm{~A}_{i}^{\omega}+\Delta+\mathrm{b}-\mathrm{AC} \vdash \tilde{\Delta}$.

For our applications in the next paragraph we need the following corollary of theorem 28:

Corollary 213 Let $\forall x^{0} \exists y^{0} \forall z^{0} A_{0}\left(u^{1}, v^{\tau}, x, y, z\right) \in \mathcal{L}\left(G_{n} A^{\omega}\right)$ be a formula which contains only $u, v$ as free variables and satisfies provably in

$G_{n} A^{\omega}+\Delta+A C-q f$ the following monotonicity property:

$(*) \forall u, v, x, \tilde{x}, y, \tilde{y}\left(\tilde{x} \leq_{0} x \wedge \tilde{y} \geq_{0} y \wedge \forall z^{0} A_{0}(u, v, x, y, z) \rightarrow \forall z^{0} A_{0}(u, v, \tilde{x}, \tilde{y}, z)\right)$,

(i.e. $\left.\operatorname{Mon}\left(\exists x \forall y \exists z \neg A_{0}\right)\right)$. Furthermore let $B_{0}\left(u, v, w^{\gamma}\right) \in \mathcal{L}\left(G_{n} A^{\omega}\right)$ be a

(quantifier-free) formula which contains only $u, v, w$ as free variables and $\gamma \leq 2$, then the following rule holds:

$$
\left\{\begin{array}{l}
\text { From a proof } \\
G_{n} A^{\omega}+\Delta+A C-q f \vdash \\
\quad \forall u^{1} \forall v \leq_{\tau} t u\left(\exists f^{1} \forall x, z A_{0}(u, v, x, f x, z) \rightarrow \exists w^{\gamma} B_{0}(u, v, w)\right) \wedge(*) \\
\text { one can extract a term } \chi \in G_{n} R^{\omega} \text { such that } \\
G_{n} A_{i}^{\omega}+\Delta+b-A C \vdash \forall u^{1} \forall v \leq_{\tau} t u \forall \Psi^{*}\left(\left(\Psi^{*}\right.\right. \text { satisfies the mon.funct.interpr. } \\
\left.\left.\quad \text { of } \forall x^{0}, g^{1} \exists y^{0} A_{0}(u, v, x, y, g y)\right) \rightarrow \exists w \leq_{\gamma} \chi u \Psi^{*} B_{0}(u, v, w)\right)^{7} .
\end{array}\right.
$$

Proof: We may assume that $\gamma=2$. The property $\operatorname{Mon}(G)$ for

$$
G: \equiv \forall u^{1} \forall v \leq_{\tau} t u \exists x^{0} \forall y^{0} \exists z^{0}, w^{2}\left(A_{0}(u, v, x, y, z) \rightarrow B_{0}(u, v, w)\right)
$$

follows logically from the monotonicity assumption $(*)$. By the assumption of the rule to be proved we have

$$
\mathrm{G}_{n} \mathrm{~A}^{\omega}+\Delta+\mathrm{AC}-\mathrm{qf} \vdash G^{H}+\operatorname{Mon}(G) .
$$

\footnotetext{
7 ' $\Psi^{*}$ satisfies the mon. funct.interpr. of $\forall x, g \exists y A_{0}(u, v, x, y, g y)$ ' is meant here for fixed $u, v$ (and not uniformly as a functional in $u, v)$, i.e. $\exists \Psi\left(\Psi^{*} \mathrm{~s}-\mathrm{maj} \Psi \wedge \forall x, g A_{0}(u, v, x, \Psi x g, g(\Psi x g))\right)$.
} 
¿From this we conclude by thm.28 that

$$
\mathrm{G}_{n} \mathrm{~A}_{i}^{\omega}+\Delta+\mathrm{b}-\mathrm{AC} \vdash \underline{\tilde{\chi}} \text { satisfies the monotone functional interpretation of } G^{\prime} \text {, }
$$

for a suitable tuple $\underline{\tilde{\chi}}$ of terms $\in \mathrm{G}_{n} \mathrm{R}^{\omega}$ which can be extracted from the proof.

$G^{\prime}$ is intuitionistically equivalent to (using the fact that $\mathrm{G}_{n} \mathrm{~A}_{i}^{\omega} \vdash \neg \neg A_{0} \leftrightarrow A_{0}$ for quantifierfree formulas $A_{0}$ )

$$
\forall u \forall v \leq t u \neg \neg \exists x^{0} \forall y^{0} \neg \neg \exists z, w\left(A_{0} \rightarrow B_{0}\right)
$$

of $G$ (This follows immediately if one uses the negative translation which is denoted by $*$ in [15] ). By intuitionistic logic the following implication holds

$$
G^{\prime} \rightarrow \forall u \forall v \leq_{\tau} t u\left(\forall x \neg \neg \exists y \forall z A_{0}(u, v, x, y, z) \rightarrow \neg \neg \exists w B_{0}(u, v, w)\right) .
$$

Hence from $\tilde{\chi}$ we obtain a term which satisfies the monotone functional interpretation of the right side of this implication. In particular we obtain a term $\hat{\chi} \in \mathrm{G}_{n} \mathrm{R}^{\omega}$ such that

$$
\begin{aligned}
\mathrm{G}_{n} \mathrm{~A}_{i}^{\omega}+\Delta+\mathrm{b}-\mathrm{AC} \vdash \exists W(\widehat{\chi} \mathrm{s}-\text { maj } W \wedge \forall u \forall v \leq t u \forall \Psi \\
\left.\left(\forall x, g A_{0}(u, v, x, \Psi x g, g(\Psi x g)) \rightarrow B_{0}(u, v, W u v \Psi)\right)\right) .
\end{aligned}
$$

Define $\chi \in \mathrm{G}_{n} \mathrm{R}^{\omega}$ by $\chi:=\lambda u^{1}, \Psi, y^{1} \cdot \widehat{\chi} u^{M}\left(t^{*} u^{M}\right) \Psi y^{M}$, where $t^{*} \in \mathrm{G}_{n} \mathrm{R}^{\omega}$ is such that $\mathrm{G}_{n} \mathrm{~A}_{i}^{\omega}$ $\vdash t^{*} \mathrm{~s}-\mathrm{maj} t$ and $u^{M}:=\lambda x^{0} \cdot \max _{i \leq x}(u i)$. Then

$$
\begin{aligned}
& \forall u \forall v \leq t u \forall \Psi^{*}\left(\exists \Psi\left(\Psi^{*} \text { s-maj } \Psi \wedge \forall x, g A_{0}(u, v, x, \Psi x g, g(\Psi x g))\right)\right. \\
& \left.\rightarrow \exists w \leq_{2} \chi u \Psi^{*} B_{0}(u, v, w)\right),
\end{aligned}
$$

since $\widehat{\chi}$ s-maj $W$ and $\Psi^{*}$ s-maj $\Psi$ imply $\forall u \forall v \leq t u\left(\chi u \Psi^{*} \geq_{2} W u v \Psi\right)$.

Remark 214 At the end of the next paragraph we will show that cor.213 does not hold for $P R A^{\omega}, \widehat{P R}^{\omega}, P R A_{i}^{\omega}$ (or $G_{n} A^{\omega}+\Sigma_{1}^{0}-I A, \widehat{P R}^{\omega}, G_{n} A_{i}^{\omega}+\Sigma_{1}^{0}-I A$ ) instead of $G_{n} A^{\omega}, G_{n} R^{\omega}, G_{n} A_{i}^{\omega}$ (even for $\Delta=\emptyset$ ). Since the proof of cor.213 from thm.28 as well as the proof of thm.28 from thm.27 extends to these theories it follows that also the theorems 27 and 28 do not hold for them. The proof of thm.27 fails for $\Psi_{i} \in \widehat{P R}^{\omega}$ since $\widehat{P R}^{\omega}$ contains functionals like $\Phi_{i t}$ which are not majorizable with finite support (see also remark 210). The proof of thm.28 fails for $G_{n} A^{\omega}+\Sigma_{1}^{0}-I A$ since the (monotone) functional interpretation of $\Sigma_{1}^{0}-I A$ requires $\Phi_{i t}$ and thus thm. 27 is not applicable.

The mathematical significance of corollary 213 for the growth of bounds extractable from given proofs rests on the following fact: Direct monotone functional interpretation of

$$
\mathrm{G}_{n} \mathrm{~A}^{\omega}+\Delta+\mathrm{AC} \text {-qf } \vdash \forall u^{1} \forall v \leq_{\tau} t u\left(\exists f^{1} \forall x, z A_{0}(u, v, x, f x, z) \rightarrow \exists w^{\gamma} B_{0}(u, v, w)\right)
$$

yields only a bound on $\exists w$ which depends on a functional which satisfies the monotone functional interpretation of (1) $\exists f \forall x, z A_{0}$ or if we let remain the double negation in front of $\exists$ (which comes from the negative translation) (2) $\neg \neg \exists f \forall x, z A_{0}$. However in our applications the monotone functional interpretation of (1) would require non-computable functionals 
(since $f$ is not recursive) and the monotone functional interpretation of (2) can be carried out only using bar-recursive functionals. In contrast to this the bound $\chi$ only depends on a functional which satisfies the monotone functional interpretation of the negative translation of $\forall x \exists y \forall z A_{0}(x, y, z)$ : In our applications such a functional can be constructed in $\widehat{P R}^{\omega}$. In particular the use of the analytical premise

$$
\exists f^{1} \forall x, z A_{0}
$$

has been reduced to the arithmetical premise

$$
\forall x^{0} \exists y^{0} \forall z^{0} A_{0} .
$$

\section{The rate of growth caused by sequences of instances of arithmetical comprehension and choice for $\Pi_{1}^{0}$-formulas}

Using the results from the previous paragraph combined with the methods from [14] one can determine the impact on the rate of growth of uniform bounds for provable $\forall u^{1} \forall v \leq_{\tau}$ $t u \exists w^{\gamma} A_{0}$-sentences which may result from the use of sequences of instances (which may depend on the parameters of the proposition to be proved) of:

1. $\Pi_{1}^{0}-\mathrm{CA}$ and $\Pi_{1}^{0}-\mathrm{AC}$.

2. The convergence of bounded monotone sequences of real numbers (PCM).

3. The existence of a greatest lower bound for every sequence of real numbers which is bounded from below.

4. The Bolzano-Weierstraß property for bounded sequences in $\mathbb{R}^{d}$ (for every fixed $d$ ).

5. The Arzelà-Ascoli lemma.

6. The existence of limsup and liminf for bounded sequences in $\mathbb{R}$.

In this paper we only consider $\Pi_{1}^{0}-\mathrm{CA}$ and $\Pi_{1}^{0}-\mathrm{AC}$ as well as certain arithmetical consequences of these principles. The treatment of the other analytical principles listed above needs a careful representation of analytical objects like continuous functions in $\mathrm{G}_{n} \mathrm{~A}^{\omega}$ as well as -in the case of 4),5)- the 'non-standard' axiom $F^{-}$introduced in [14] and will be carried out in a subsequent paper.

Definition $31 \Pi_{1}^{0}-C A\left(f^{1(0)}\right): \equiv \exists g^{1} \forall x^{0}\left(g x={ }_{0} 0 \leftrightarrow \forall y^{0}\left(f x y={ }_{0} 0\right)\right)$.

(Note that iteration of $\forall f^{1(0)}\left(\Pi_{1}^{0}-\mathrm{CA}(f)\right)$ yields $\left.\mathrm{CA}_{a r}\right)$.

\section{Definition 32}

Define $A_{0}^{C}\left(f^{1(0)}, x^{0}, y^{0}, z^{0}\right): \equiv \forall \tilde{x} \leq_{0} x \exists \tilde{y} \leq_{0} y \forall \tilde{z} \leq_{0} z\left(f \tilde{x} \tilde{y} \neq_{0} 0 \vee f \tilde{x} \tilde{z}=_{0} 0\right)$.

$A_{0}^{C}$ can be expressed as a quantifier-free formula in $G_{n} A^{\omega}$ (see [14]).

Proposition 33 For $n \geq 1$ one has:

1. $G_{n} A_{i}^{\omega}$ proves

$$
\forall f, x, \tilde{x}, y, \tilde{y}\left(\tilde{x} \leq_{0} x \wedge \tilde{y} \geq_{0} y \wedge \forall z^{0} A_{0}^{C}(f, x, y, z) \rightarrow \forall z^{0} A_{0}^{C}(f, \tilde{x}, \tilde{y}, z)\right) .
$$


2. $G_{n} A_{i}^{\omega} \vdash \forall f^{1(0)}\left(\exists g^{1} \forall x^{0}, z^{0} A_{0}^{C}(f, x, g x, z) \rightarrow \Pi_{1}^{0}-C A(f)\right)$.

3. For the functional $\Phi \in \widehat{P R}^{\omega}$ defined by $\Phi x^{0} h^{1}:=\max _{i \leq x+1} h^{i}(0)$ we have

$$
P R A_{i}^{\omega} \vdash \Phi s-m a j \Phi \wedge \forall f^{1(0)}, x^{0}, h^{1} \exists y \leq{ }_{0} \Phi x h A_{0}^{C}(f, x, y, h y) .
$$

Hence $\Phi$ satisfies (provably in $P R A_{i}^{\omega}$ ) the monotone functional interpretation of $\forall x, h \exists y A_{0}^{C}(f, x, y, h y)$ for all $f^{1(0)}$.

Proof: 1) is obvious.

2) Let $g$ be such that $\forall x, z \forall \tilde{x} \leq x \exists \tilde{y} \leq g x \forall \tilde{z} \leq z(f \tilde{x} \tilde{y} \neq 0 \vee f \tilde{x} \tilde{z}=0)$. By taking $\tilde{x}:=x$ and $\tilde{z}:=z$ we obtain $\forall x, z \exists \tilde{y} \leq g x(f x \tilde{y} \neq 0 \vee f x z=0)$ and thus

$$
\forall x(\forall \tilde{y} \leq g x(f x \tilde{y}=0) \leftrightarrow \forall z(f x z=0)) .
$$

Hence $h x:=\left\{\begin{array}{l}0, \text { if } \forall \tilde{y} \leq g x(f x \tilde{y}=0) \\ 1, \text { otherwise }\end{array}\right.$ satisfies $\Pi_{1}^{0}-\mathrm{CA}(f)$.

3) Assume that

(*) $\forall y \leq \Phi x h \exists \tilde{x} \leq x \forall \tilde{y} \leq y \exists \tilde{z} \leq h y(f \tilde{x} \tilde{y}=0 \wedge f \tilde{x} \tilde{z} \neq 0)$.

Case 1: $\exists i<x+1\left(h\left(h^{i} 0\right) \leq h^{i} 0\right)$ :

$(*)$ applied to $y:=h^{i} 0 \leq \Phi x h$ yields an $\tilde{x} \leq x$ such that

$$
(* *) \forall \tilde{y} \leq h^{i} 0 \exists \tilde{z} \leq h\left(h^{i} 0\right)(f \tilde{x} \tilde{y}=0 \wedge f \tilde{x} \tilde{z} \neq 0)
$$

and thus for $\tilde{y}:=0$ one has a $\tilde{z} \leq h\left(h^{i} 0\right)$ such that $f \tilde{x} \tilde{z} \neq 0$. But on the other hand -again by $(* *)$ - one has $f \tilde{x} \tilde{z}=0$ (since $\left.\tilde{z} \leq h\left(h^{i} 0\right) \leq h^{i} 0\right)$ which is a contradiction.

Case 2: $\forall i<x+1\left(h\left(h^{i} 0\right)>h^{i} 0\right)$ : that

By the pigeon-hole principle, $(*)$ implies that there exists $i<j \leq x+1$ and $\tilde{x} \leq x$ such

$$
\text { (1) } \forall \tilde{y} \leq h^{i} 0 \exists \tilde{z} \leq h\left(h^{i} 0\right)(f \tilde{x} \tilde{y}=0 \wedge f \tilde{x} \tilde{z} \neq 0)
$$

and

$$
\text { (2) } \forall \tilde{y} \leq h^{j} 0 \exists \tilde{z} \leq h\left(h^{j} 0\right)(f \tilde{x} \tilde{y}=0 \wedge f \tilde{x} \tilde{z} \neq 0) \text {. }
$$

Hence $\exists \tilde{z} \leq h\left(h^{i} 0\right)(f \tilde{x} \tilde{z} \neq 0)$ by (1) (take $\left.\tilde{y}:=0\right)$ and $\forall \tilde{y} \leq h^{j} 0(f \tilde{x} \tilde{y}=0)$ by (2) which is a contradiction since by the case (and $i<j \leq x+1) h\left(h^{i} 0\right)=h^{i+1} 0 \leq h^{j} 0$.

Put together we have proved that $\forall f, x, h \exists y \leq_{0} \Phi x h A_{0}^{C}(f, x, y, h y)$ which is equivalent to a purely universal sentence and hence an axiom of $\mathrm{G}_{n} \mathrm{~A}_{i}^{\omega}$ (In fact one easily verifies that this assertion would also be provable in $G_{n} A_{i}^{\omega}$ if we would have instead of the universal axioms only the schema of quantifier-free induction included as axioms of $\mathrm{G}_{n} \mathrm{~A}_{i}^{\omega}$ ).

It remains to show that $\Phi \mathrm{s}-$ maj $\Phi$ : Assume that $\tilde{h} \mathrm{~s}-\operatorname{maj}_{1} h$. By quantifier-free induction on $x$ one shows that $\forall x\left(\tilde{h}^{x} 0 \geq h^{x} 0\right)$. Hence (by quantifier-free induction on $\left.\tilde{x}\right): \forall \tilde{x}, x(\tilde{x} \geq$ $x \rightarrow \Phi \tilde{x} \tilde{h} \geq \Phi x h)$.

Cor.213 combined with prop.33 yields 
Proposition 34 Let $n \geq 1$ and $B_{0}\left(u^{1}, v^{\tau}, w^{\gamma}\right) \in \mathcal{L}\left(G_{n} A^{\omega}\right)$ be a quantifier-free formula which contains only $u^{1}, v^{\tau}, w^{\gamma}$ free, where $\gamma \leq 2$. Furthermore let $\xi, t \in G_{n} R^{\omega}$ and $\Delta$ be as in thm.28. Then the following rule holds

$$
\left\{\begin{array}{l}
G_{n} A^{\omega}+\Delta+A C-q f \vdash \forall u^{1} \forall v \leq_{\tau} t u\left(\Pi_{1}^{0}-C A(\xi u v) \rightarrow \exists w^{\gamma} B_{0}(u, v, w)\right) \\
\Rightarrow \exists(e f f .) \chi \in G_{n} R^{\omega} \text { such that } \\
G_{n} A_{i}^{\omega}+\Delta+b-A C \vdash \forall u^{1} \forall v \leq_{\tau} t u \forall \Psi^{*}\left(\left(\Psi^{*}\right.\right. \text { satifies the mon. funct.interpr. } \\
\left.\left.\quad \text { of } \forall x^{0}, h^{1} \exists y^{0} A_{0}^{C}(\xi u v, x, y, h y)\right) \rightarrow \exists w \leq_{\gamma} \chi u \Psi^{*} B_{0}(u, v, w)\right) \\
\text { and in particular } \quad P R A_{i}^{\omega}+\Delta+b-A C \vdash \forall u^{1} \forall v \leq_{\tau} t u \exists w \leq_{\gamma} \chi u \Psi B_{0}(u, v, w),
\end{array}\right.
$$

where $\Psi:=\lambda x^{0}, h^{1} \cdot \max _{i<x+1}\left(\Phi_{i t} i 0 h\right)\left(=\lambda x^{0}, h^{1} \cdot \max _{i<x+1}\left(h^{i} 0\right)\right)$.

In the conclusion, $\Delta+b-A C$ can be replaced by $\tilde{\Delta}$, where $\tilde{\Delta}$ is defined as in thm.28. If $\Delta=\emptyset$, then $b-A C$ can be omitted from the proof of the conclusion. If $\tau \leq 1$ and the types of the $\exists$-quantifiers in $\Delta$ are $\leq 1$, then $G_{n} A^{\omega}+\Delta+A C-q f$ may be replaced by $E-G_{n} A^{\omega}+\Delta+A C^{\alpha, \beta}{ }_{-}$ qf, where $\alpha, \beta$ are as in cor.211.

Remark 35 In general prop.34 only guarantees a primitive recursive (in the sense of Kleene $[6],[7]$ and not only in the generalized sense of Gödel's T) bound $\Phi:=\lambda u \cdot \chi u \Psi \in \widehat{P R}^{\omega}$ on $\exists w$. This is not avoidable since $\Pi_{1}^{0}-C A(\xi(f))$ proves $\Sigma_{1}^{0}-I A(f)$ relative to $G_{n} A^{\omega}$ for suitable $\xi$. If however the proof applies $\Psi$ only to special functions like e.g. $h:=S$ then much better bounds will result.

\section{We now consider $\Pi_{1}^{0}$-instances of $\mathrm{AC}_{a r}$ :}

$$
\Pi_{1}^{0}-\mathrm{AC}\left(f^{1(0)(0)(0)}\right): \equiv \forall l^{0}\left(\forall x^{0} \exists y^{0} \forall z^{0}\left(f l x y z={ }_{0} 0\right) \rightarrow \exists g^{1} \forall x^{0}, z^{0}\left(f l x(g x) z={ }_{0} 0\right)\right) .
$$

$\Pi_{1}^{0}-\mathrm{AC}(f)$ can be reduced to $\Pi_{1}^{0}-\mathrm{CA}(g)$ uniformly by

\section{Proposition 36}

$$
G_{2} A^{\omega}+A C^{0,0}-q f \vdash \forall f^{1(0)(0)(0)}\left(\Pi_{1}^{0}-C A\left(f^{\prime}\right) \rightarrow \Pi_{1}^{0}-A C(f)\right),
$$

where $f^{\prime}:=\lambda v^{0}, z^{0} \cdot f\left(\nu_{1}^{3}(v), \nu_{2}^{3}(v), \nu_{3}^{3}(v), z\right)^{8}$.

Proof: By $\Pi_{1}^{0}-\mathrm{CA}\left(f^{\prime}\right)$ there exists a function $h^{1}$ such that

$$
\forall v^{0}\left(h v=0 \leftrightarrow \forall z\left(f^{\prime} v z=0\right)\right) .
$$

$\tilde{h} l x y:=h\left(\nu^{3}(l, x, y)\right)$. Then

$$
\forall l, x, y(\tilde{h} l x y=0 \leftrightarrow \forall z(f l x y z=0)) .
$$

$\mathrm{AC}^{0,0}$-qf applied to $\forall x \exists y(\tilde{h} l x y=0)$ yields $\exists g \forall x, z(f l x(g x) z=0)$.

As a corollary of prop.34 and prop.36 we obtain

\footnotetext{
${ }^{8}$ Here $\nu_{i}^{k}$ are the coding functions for tuples from [14].
} 
Corollary 37 For $n \geq 2$ proposition 34 also holds with $\Pi_{1}^{0}-A C(\xi u v)$ (but now with $A_{0}^{C}\left((\xi u v)^{\prime}, x, y, h y\right)$ in the conclusion).

Remark 38 Suppose that $n \geq 2$.

1. We may also have finite conjunctions $\bigwedge_{i=1}^{l} \Pi_{1}^{0}-C A\left(\xi_{i} u v\right) \wedge \bigwedge_{i=1}^{j} \Pi_{1}^{0}-A C\left(\tilde{\xi}_{i} u v\right)$ of instances of $\Pi_{1}^{0}-C A$ and $\Pi_{1}^{0}-A C$ in prop. 34 (with a suitable $\tilde{\xi} \in G_{n} R^{\omega}$ instead of $\xi$ in the conclusion): Since instances of $\Pi_{1}^{0}-A C$ reduce to instances of $\Pi_{1}^{0}-C A$ by the proposition above we only have to verify (in $G_{2} A^{\omega}$ ) that $\Pi_{1}^{0}-C A(f) \wedge \Pi_{1}^{0}-C A(g) \rightarrow \Pi_{1}^{0}-C A(\varphi f g)$, where $\varphi \in G_{2} R^{\omega}$ is defined by $\varphi f g x y:= \begin{cases}f\left(j_{2} x, y\right), & \text { if } j_{1} x=0 \\ g\left(j_{2} x, y\right), & \text { otherwise. }\end{cases}$

This however is clear.

2. In prop.34 even sequences $\forall l^{0} \Pi_{1}^{0}-C A(\xi u v l), \forall l^{0} \Pi_{1}^{0}-A C(\xi u v l)$ of instances of $\Pi_{1}^{0}-C A, \Pi_{1}^{0}$ $A C$ are allowed (instead of $\Pi_{1}^{0}-C A(\xi u v), \Pi_{1}^{0}-A C(\xi u v)$ only) since such sequences of instances can be reduced to single instances in $G_{2} A^{\omega}: \forall l^{0} \Pi_{1}^{0}-C A(f l)$ follows from $\Pi_{1}^{0}$ $C A\left(f^{\prime}\right)$, where $f^{\prime} x y:=f\left(j_{1} x, j_{2} x, y\right)$. Similarly for $\Pi_{1}^{0}-A C$ (note that the universal closure under arithmetical parameters has already been incorporated within the definition of $\Pi_{1}^{0}$ $A C(f))$.

By $\Pi_{1}^{0}-\mathrm{CA}^{-}, \Pi_{1}^{0}-\mathrm{AC}^{-}$and $\Sigma_{2}^{0}-\mathrm{AC}^{-}$we denote the schemas of $\Pi_{1}^{0}$-comprehension and $\Pi_{1}^{0}, \Sigma_{2}^{0}-$ choice for formulas without parameters of type $\geq 1$, i.e.

$$
\begin{gathered}
\Pi_{1}^{0}-\mathrm{CA}^{-}: \exists f \forall x^{0}\left(f x={ }_{0} 0 \leftrightarrow \forall y^{0} A_{0}\left(x, y, \underline{a}^{0}\right)\right), \\
\Pi_{1}^{0}-\mathrm{AC}^{-}: \forall x^{0} \exists y^{0} \forall z^{0} A_{0}\left(x, y, z, \underline{a}^{0}\right) \rightarrow \exists f \forall x \forall z A_{0}(x, f x, z, \underline{a}), \\
\Sigma_{2}^{0}-\mathrm{AC}^{-}: \quad \forall x^{0} \exists y^{0} \exists z^{0} \forall v^{0} A_{0}\left(x, y, z, v, \underline{a}^{0}\right) \rightarrow \exists f \forall x \exists z \forall v A_{0}(x, f x, z, v, \underline{a}),
\end{gathered}
$$

where only $x, y, \underline{a}(x, y, z, \underline{a}$ resp. $x, y, z, v, \underline{a})$ occur free in $A_{0}(x, y, \underline{a})\left(A_{0}(x, y, z, \underline{a})\right.$ resp. $\left.A_{0}(x, y, z, v, \underline{a})\right)$.

As a special case of prop.34 and cor.37 we have

Proposition 39 Let $n \geq 2$ and $\gamma \leq 2$ and $B_{0}\left(u^{1}, v^{\tau}, w^{\gamma}\right)$ contains only $u, v, w$ as free variables; $t \in G_{n} R^{\omega}$. Then the following rule holds

$$
\left\{\begin{array}{l}
G_{n} A^{\omega} \oplus A C-q f \oplus \Pi_{1}^{0}-C A^{-} \oplus \Sigma_{2}^{0}-A C^{-} \vdash \forall u^{1} \forall v \leq_{\tau} t u \exists w^{\gamma} B_{0}(u, v, w) \\
\Rightarrow \exists \Psi \in \widehat{P R} \text { such that } \\
P R A_{i}^{\omega} \vdash \forall u^{1} \forall v \leq_{\tau} t u \exists w \leq_{\gamma} \Psi u B_{0}(u, v, w) .
\end{array}\right.
$$

If $\tau \leq 1$, we may replace $G_{n} A^{\omega} \oplus A C-q f \oplus \Pi_{1}^{0}-C A^{-} \oplus \Sigma_{2}^{0}-A C^{-}$by

$$
\begin{aligned}
& E-G_{n} A^{\omega}+A C^{\alpha, \beta}-q f+\Pi_{1}^{0}-C A^{-}+\Sigma_{2}^{0}-A C^{-}, \text {where }(\alpha=0 \wedge \beta \leq 1) \text { or } \\
& (\alpha=1 \wedge \beta=0) .
\end{aligned}
$$


In particular

$$
\left\{\begin{array}{l}
E-G_{n} A^{\omega}+A C^{\alpha, \beta}-q f+\Pi_{1}^{0}-C A^{-}+\Sigma_{2}^{0}-A C^{-} \vdash \forall u^{0} \exists v^{0} R(u, v) \\
\Rightarrow \exists \text { primitive recursive function } \varphi: \\
\forall u R(u, \varphi u) \text { is true, }
\end{array}\right.
$$

where $R$ is a primitive recursive relation. If in the definition of $G_{n} A^{\omega}$ the universal axioms 9) are replaced by the schema of quantifier-free induction one has $P R A \vdash R(u, \varphi u)$

(Note that this proposition also holds for $n=\infty$. Since all primitive recursive functions (but not all primitive recursive functionals of type 2!) can be defined in $G_{\infty} A^{\omega}$ (see §2 of [14]) we may assume that $\left.G_{\infty} A^{\omega} \supset P R A\right)$.

Proof: Since $\Pi_{1}^{0}-\mathrm{CA}^{-}$follows from $\Sigma_{2}^{0}-\mathrm{AC}^{-}$which in turn is implied by $\Pi_{1}^{0}-\mathrm{AC}^{-}$(using pairing) it suffices to consider an instance $A$ of the later (for simplicity we may assume that we have only one arithmetical parameter):

$$
A\left(a^{0}\right): \equiv \forall x^{0} \exists y^{0} \forall z^{0} A_{0}(x, y, z, a) \rightarrow \exists f^{1} \forall x, z A_{0}(x, f x, z, a),
$$

where $A_{0}(x, y, z, a)$ is quantifier-free and contains only $x, y, z, a$ as free variables. Let $\xi \in \mathrm{G}_{n} \mathrm{R}^{\omega}$ be such that $\xi a x y z={ }_{0} 0 \leftrightarrow A_{0}(x, y, z, a)$. Then $\Pi_{1}^{0}-\mathrm{AC}(\xi)$ implies $\forall a^{0} A(a)$. The corollary now follows from prop.34, cor.37 and rem.38.1) by the deduction theorem for $\oplus$.

Remark 310 Even for the second-order fragment $\mathrm{G}_{2} \mathrm{~A}^{2}$ of $\mathrm{G}_{2} \mathrm{~A}^{\omega}$ (and without the universal axioms 9) but only the schema of quantifier-free induction instead of them) the theory $\mathrm{G}_{2} \mathrm{~A}^{2}+\Pi_{1}^{0}-\mathrm{CA}^{-}+\Pi_{2}^{0}$-IR ${ }^{-}$proves the totality of the Ackermann function (see [13]). This refutes a result stated in Mints [16] and a fortiori various generalizations of this result stated in [19] (thm.5.8,cor.5.9,thm.5.13,cor.5.14(ii)). For details see [12](chapter 12) and also [13].

Proposotion 39 also becomes false if the primitive recursive functional $\tilde{\Phi} 0 y f={ }_{0} y, \tilde{\Phi} x^{\prime} y f={ }_{0}$ $f(x, y, \tilde{\Phi} x y f)$ is added to $\mathrm{G}_{n} \mathrm{~A}^{\omega}$ (see [12](chapter 12)). Therefore any proof of a result like this proposition has to exploit carefully the structure of the type-2-functionals of $\mathrm{G}_{n} \mathrm{~A}^{\omega}$.

\section{Arithmetical consequences of $\Pi_{1}^{0}-\mathbf{C A}(f)$ and $\Pi_{1}^{0}-\mathbf{A C}(f)$}

Using $\Pi_{1}^{0}-\mathrm{CA}(f)$ we can prove (relatively to $\mathrm{G}_{2} \mathrm{~A}^{\omega}$ ) every instance of $\Delta_{2}^{0}$-IA with fixed function parameters:

$$
\Delta_{2}^{0}-\mathrm{IA}(f, g): \equiv\left\{\begin{array}{c}
\forall l^{0}\left(\forall x^{0}\left(\exists u^{0} \forall v^{0}\left(f l x u v==_{0} 0\right) \leftrightarrow \forall \tilde{u}^{0} \exists \tilde{v}^{0}\left(g l x \tilde{u} \tilde{v}={ }_{0} 0\right)\right) \rightarrow\right. \\
{\left[\exists u \forall(f l 0 u v=0) \wedge \forall x\left(\exists u \forall v(f l x u v=0) \rightarrow \exists u \forall v\left(f l x^{\prime} u v=0\right)\right)\right.} \\
\rightarrow \forall x \exists u \forall v(f l x u v=0)]) .
\end{array}\right.
$$

Define $f^{\prime}:=\lambda i^{0}, v^{0} \cdot f\left(\nu_{1}^{3}(i), \nu_{2}^{3}(i), \nu_{3}^{3}(i), v\right)$ and

$g^{\prime}:=\lambda i^{0}, v^{0} \cdot \overline{s g}\left(g\left(\nu_{1}^{3}(i), \nu_{2}^{3}(i), \nu_{3}^{3}(i), v\right)\right)$. We now show

\section{Proposition 311}

$$
G_{2} A^{\omega}+A C^{0,0}-q f \vdash \forall f, g\left(\Pi_{1}^{0}-C A\left(f^{\prime}\right) \wedge \Pi_{1}^{0}-C A\left(g^{\prime}\right) \rightarrow \Delta_{2}^{0}-I A(f, g)\right) .
$$


Proof: $\Pi_{1}^{0}-\mathrm{CA}\left(f^{\prime}\right)$ and $\Pi_{1}^{0}-\mathrm{CA}\left(g^{\prime}\right)$ imply the existence of functions $h_{1}, h_{2}$ such that for all $l, x, u$

$$
h_{1} l x u={ }_{0} 0 \leftrightarrow \forall v\left(f l x u v={ }_{0} 0\right) \text { and } h_{2} l x u={ }_{0} 0 \leftrightarrow \exists v\left(g l x u v={ }_{0} 0\right) .
$$

Assume now that

$$
\forall x^{0}\left(\exists u^{0} \forall v^{0}\left(f l x u v={ }_{0} 0\right) \leftrightarrow \forall \tilde{u}^{0} \exists \tilde{v}^{0}\left(g l x \tilde{u} \tilde{v}={ }_{0} 0\right)\right) .
$$

Then

$$
\forall x\left(\exists u\left(h_{1} l x u=0\right) \leftrightarrow \forall \tilde{u}\left(h_{2} l x \tilde{u}=0\right)\right) .
$$

With classical logic this yields

$$
\forall x^{0} \exists z^{0}(\underbrace{\left[\forall \tilde{u}\left(h_{2} l x \tilde{u}=0\right) \rightarrow z=0\right] \wedge\left[z=0 \rightarrow \exists u\left(h_{1} l x u=0\right)\right]}_{\in \Sigma_{1}^{0}}) .
$$

By $\mathrm{AC}^{0,0}-$ qf we obtain a function $\alpha$ such that

$$
\forall x\left(\alpha x=0 \leftrightarrow \exists u\left(h_{1} l x u=0\right)\right) .
$$

$\Delta_{2}^{0}-\mathrm{IA}(f, g)$ now follows by applying QF-IA to $A_{0}(x): \equiv(\alpha x=0)$.

Next we show that $\Pi_{1}^{0}$-instances (with fixed function parameters) of the collection principle

$$
\mathrm{CP}: \forall \tilde{x}<_{0} x \exists y^{0} A(x, \tilde{x}, y) \rightarrow \exists y_{0} \forall \tilde{x}<_{0} x \exists y<_{0} y_{0} A(x, \tilde{x}, y) .
$$

are derivable from $\Pi_{1}^{0}-\mathrm{AC}$-instances.

$$
\begin{aligned}
& \Pi_{1}^{0}-\mathrm{CP}(f): \equiv \\
& \forall l^{0}, x^{0}\left(\forall \tilde{x}<x \exists y^{0} \forall z^{0}\left(f l x \tilde{x} y z={ }_{0} 0\right) \rightarrow \exists y_{0} \forall \tilde{x}<x \exists y<<_{0} y_{0} \forall z(f l x \tilde{x} y z=0)\right) .
\end{aligned}
$$

\section{Proposition 312}

$$
G_{2} A^{\omega} \vdash \forall f\left(\Pi_{1}^{0}-A C\left(f^{\prime}\right) \rightarrow \Pi_{1}^{0}-C P(f)\right),
$$

where $f^{\prime}$ such that $f^{\prime} i \tilde{x} y z={ }_{0} 0 \leftrightarrow\left(\tilde{x}<\nu_{2}^{2}(i) \rightarrow f\left(\nu_{1}^{2}(i), \nu_{2}^{2}(i), \tilde{x}, y, z\right)={ }_{0} 0\right)$.

Proof: $\Pi_{1}^{0}-\mathrm{AC}\left(f^{\prime}\right)$ yields

$$
\forall l^{0}, x^{0}\left(\forall \tilde{x}<x \exists y \forall z(f l x \tilde{x} y z=0) \rightarrow \exists h^{1} \forall \tilde{x}<x \forall z(f l x \tilde{x}(h \tilde{x}) z=0)\right) .
$$

Define $y_{0}:=1+\Phi_{1} h x$ (Recall that $\left.\Phi_{1} h x:=\max _{i \leq x}(h i)\right)$.

We conclude this paper by showing that cor.213 is false (even for $\Delta=\emptyset$ ) when $\mathrm{G}_{n} \mathrm{~A}^{\omega}$, $\mathrm{G}_{n} \mathrm{R}^{\omega}, \mathrm{G}_{n} \mathrm{~A}_{i}^{\omega}$ are replaced by $\mathrm{G}_{n} \mathrm{~A}^{\omega}+\Sigma_{1}^{0}-\mathrm{IA}, \widehat{P R}^{\omega}, \mathrm{G}_{n} \mathrm{~A}_{i}^{\omega}+\Sigma_{1}^{0}-\mathrm{IA}$ or PRA ${ }^{\omega}, \widehat{P R}^{\omega}, \mathrm{PRA}_{i}^{\omega}$ : It is well-known that there is an (function parameter-free) instance $G$ of $\Pi_{2}^{0}$-IA such that

$$
\mathrm{G}_{3} \mathrm{~A}^{\omega}+\Sigma_{1}^{0}-\mathrm{IA}+G \vdash \forall x^{0} \exists y^{0} A_{0}(x, y),
$$


where $\forall x \exists y \leq f x A_{0}(x, y)$ implies that $f$ has the growth of the Ackermann function.

Let $B\left(x^{0}\right): \equiv \forall u^{0} \exists v^{0} B_{0}\left(a^{0}, u, v, x\right)$ be the induction formula of $G$, where $B_{0}(a, u, v, x)$ contains only $a, u, v, x$ as free variables. By applying $\Pi_{1}^{0}-\mathrm{CA}(f)$ to $f(i, v):=t_{B_{0}}\left(\nu_{1}^{3}(i), \nu_{2}^{3}(i), v, \nu_{3}^{3}(i)\right)$, where $t_{B_{0}}$ is the characteristic function of $B_{0}, G$ reduces to $\Pi_{1}^{0}$-IA (with function parameters) and hence to $\Sigma_{1}^{0}$-IA $\left(\Pi_{n}^{0}-\mathrm{IA}\right.$ and $\Sigma_{n}^{0}$-IA are equivalent, see [19]). Hence

$$
\mathrm{G}_{3} \mathrm{~A}^{\omega}+\Sigma_{1}^{0}-\mathrm{IA} \vdash \Pi_{1}^{0}-\mathrm{CA}(f) \rightarrow \forall x \exists y A_{0}(x, y) .
$$

If cor.213 would apply to $\mathrm{G}_{3} \mathrm{~A}^{\omega}+\Sigma_{1}^{0}$-IA and $\widehat{P R}^{\omega}$ we would obtain (by the proof of prop. 34) a term $s^{1} \in \widehat{P R}^{\omega}$ such that $\forall x \exists y \leq s x A_{0}(x, y)$. This however would contradict the well-known fact that every $s^{1} \in \widehat{P R}^{\omega}$ is primitive recursive.

The same argument applies to $\mathrm{PRA}^{\omega}$ since $\mathrm{PRA}^{\omega}+\mathrm{AC}^{0,0}-\mathrm{qf} \vdash \Sigma_{1}^{0}-\mathrm{IA}$.

\section{References}

1. Feferman, S., Theories of finite type related to mathematical practice. In: Barwise, J. (ed.), Handbook of Mathematical Logic, North-Holland, Amsterdam, pp. 913-972 (1977).

2. Friedman, H., Some systems of second order arithmetic and their use. In: Proc. 1974 International Congress of Mathematicians, Vancouver 1974, vol. 1 (Canadian Mathematical Congress, 1975), pp.235-242 (1974).

3. Friedman, H., Systems of second order arithmetic with restricted induction (abstract), J. Symbolic Logic 41, pp. 558-559 (1976).

4. Gödel, K., Zur intuitionistischen Arithmetik und Zahlentheorie. Ergebnisse eines Mathematischen Kolloquiums, vol. 4 pp. 34-38 (1933).

5. Gödel, K., Über eine bisher noch nicht benutzte Erweiterung des finiten Standpunktes. Dialectica 12, pp. 280-287 (1958).

6. Kleene, S.C.,Introduction to Metamathematics. North- Holland (Amsterdam), Noordhoff (Groningen), New-York (Van Nostrand) (1952).

7. Kleene, S.C., Recursive functionals and quantifiers of finite types, I. Trans. A.M.S. 91, pp.1-52 (1959).

8. Kohlenbach, U., Effective bounds from ineffective proofs in analysis: an application of functional interpretation and majorization. J. Symbolic Logic 57, pp.1239-1273 (1992).

9. Kohlenbach, U., Pointwise hereditary majorization and some applications. Arch. Math. Logic 31, pp.227-241 (1992).

10. Kohlenbach, U., Remarks on Herbrand normal forms and Herbrand realizations. Arch. Math. Logic 31, pp.305-317 (1992).

11. Kohlenbach, U., Analysing proofs in analysis. In: W. Hodges, M. Hyland, C. Steinhorn, J. Truss, editors, Logic: from Foundations to Applications, European Logic Colloquium (Keele 1993),pp. 225-260, Clarendon Oxford (1996). Oxford University Press.

12. Kohlenbach, U., Real growth in standard parts of analysis. Habilitationsschrift, $\mathrm{xv}+166 \mathrm{pp}$., Frankfurt (1995).

13. Kohlenbach, U., A note on the $\Pi_{2}^{0}$-induction rule. Arch. Math. Logic 34, pp. 279-283 (1995).

14. Kohlenbach, U., Mathematically strong subsystems of analysis with low rate of provably recursive functionals. Arch. Math. Logic 36, pp. 31-71 (1996).

15. Luckhardt, H., Extensional Gödel functional interpretation. A consistency proof of classical analysis. Springer Lecture Notes in Mathematics 306 (1973).

16. Mints, G.E., What can be done with PRA. J.Soviet Math. 14, pp.1487-1492, 1980 (Translation from: Zapiski Nauchuyh Seminarov, LOMI,vol. 60 (1976),pp. 93-102). 
17. Parsons, C., On a number theoretic choice schema and its relation to induction. In: Intuitionism and proof theory, pp. 459-473. North-Holland, Amsterdam (1970).

18. Parsons, C., Proof-theoretic analysis of restricted induction schemata (abstract). J. Symbolic Logic 36, p.361 (1971).

19. Sieg, W., Fragments of arithmetic. Ann. Pure Appl. Logic 28, pp. 33-71 (1985).

20. Simpson, S.G., Reverse Mathematics. Proc. Symposia Pure Math. 42, pp. 461-471, AMS, Providence (1985).

21. Spector, C., Provably recursive functionals of analysis: a consistency proof of analysis by an extension of principles formulated in current intuitionistic mathematics. In: Recursive function theory, Proceedings of Symposia in Pure Mathematics, vol. 5 (J.C.E. Dekker (ed.)), AMS, Providence, R.I., pp. 1-27 (1962). 